\title{
LOW ORDER DISCONTINUOUS GALERKIN METHODS FOR SECOND ORDER ELLIPTIC PROBLEMS
}

\author{
E. BURMAN* AND B. STAMM ${ }^{\dagger}$
}

\begin{abstract}
We consider DG-methods for 2nd order scalar elliptic problems using piecewise affine approximation in two or three space dimensions. We prove that both the symmetric and the nonsymmetric version of the DG-method are well-posed also without penalization of the interelement solution jumps provided boundary conditions are imposed weakly. Optimal convergence is proved for sufficiently regular meshes and data. We then propose a discontinuous Galerkin method using piecewise affine functions enriched with quadratic bubbles. Using this space we prove optimal convergence in the energy norm for both a symmetric and non-symmetric DG-method without stabilization. All these proposed methods share the feature that they conserve mass locally independent of the penalty parameter.
\end{abstract}

Key words. Discontinuous Galerkin; elliptic equation; Crouzeix-Raviart approximation; interior penalty; local mass conservation.

AMS subject classifications. 65M160, 65M15

1. Introduction. The discontinuous Galerkin method (DG) for (2n)th-order elliptic problems was introduced and analysed by Baker [2] with special focus on the fourth order case. In this work both the jumps of the solution and its gradient were penalized. Douglas and Dupont however announced already in [10] a forthcoming analysis for discontinuous approximations and penalty on the solution jumps. This analysis was then realized in the work of Wheeler [22], in the framework of collocation methods for discontinuous Galerkin and Arnold [1] in the framework of parabolic problems leading to the symmetric interior penalty DG-method (SIPG).

During the nineties there was a strong development of discontinuous Galerkin methods for elliptic problems. Bassi and Rebay proposed a formulation for NavierStokes equations [3], introducing an auxiliary variable for the diffusive fluxes, this method was then analysed by Cockburn and co workers in $[8,7]$ and more recently in the framework of Friedrichs systems by Ern and Guermond [12].

Babuska, Baumann and Oden proposed a non-symmetric method for elliptic problems with a less stiff penalization term [17] (NIPG). This method was modified by Oden, Prudhomme and Romkes in [19] where they proposed a DG-method for reaction-diffusion equations with penalization of the diffusive fluxes to enhance stability for the non-symmetric version without perturbing the local massconservation properties. The DG-methods for second order elliptic problems have been further analysed in the works by Girault, Rivière and Wheeler [18] and Larson and Niklasson [15]. In the last reference the authors proved that in the non-symmetric case when using high order polynomial approximation the stabilization term may be dropped since control of the solution jumps can be recovered from the anti-symmetric part of the diffusion-operator using an inf-sup argument.

For a review of discontinuous Galerkin methods for elliptic problems we refer to Arnold et al. [1] and for a review of stabilization mechanisms in discontinuous Galerkin methods we refer to Brezzi et al. [5].

\footnotetext{
*Institute of Analysis and Scientific Computing, Swiss Institute of Technology, Lausanne, CH1015, Switzerland (erik.burman@epfl.ch).

${ }^{\dagger}$ Institute of Analysis and Scientific Computing, Swiss Institute of Technology, Lausanne, CH1015, Switzerland (benjamin.stamm@epfl.ch). Supported by Swiss National Foundation.
} 
One of the advantages of the discontinuous Galerkin method is that it has enhanced local conservation compared to the continuous Galerkin method. On the other hand, as was pointed out by Larson and Niklasson in [13] this local conservation is not independent of the penalization operator. Only in the case of the non-symmetric DG method without penalization of the solution jumps or with penalization on the fluxes is it possible to obtain local mass conservation independently of the penalization parameter. In both cases however the formulation is not adjoint consistent and one may not prove $L^{2}$-convergence using the Nitsche trick.

The question of how much the discontinuous Galerkin method needs to be stabilized was discussed in the case of mixed formulations of elliptic problems by Sherwin et al. in [20] and by Marazzina in [16]. It was found that stabilization needs to be applied on the boundary of the domain only to assure well-posedness of the discrete system.

In this paper we discuss the relation between stabilization, well posedness and optimal convergence in the case of scalar second order elliptic problems. The aim is to design a low order DG-method that

1. has optimal convergence in the $H^{1}$ - and $L^{2}$-norm,

2 . is locally massconservative independently of the penalty parameter.

We will show that for the symmetric DG-method the only thing required to obtain well-posedness of the discrete system is weakly imposed boundary conditions. No interelement penalization of the solution jumps is required. In this case of course local mass conservation is obtained independently of the penalty parameter. Optimal convergence is also obtained only imposing boundary conditions, however under strong assumptions on the computational mesh and data. Either the mesh has to satify as certain macro element property, or mesh and data have to be sufficiently regular. If these conditions are not satisfied the convergence of the solution jumps can be perturbed by the appearance of a checkerboard mode that vanishes too slowly in the absence of interior penalty. We exemplify the checkerboard mode numerically and show how it is quenched by penalization.

To reduce the constraints on the mesh we enrich the space with quadratic nonconforming bubble functions. These stabilizing bubbles eliminate the checkerboard mode. This allows for optimal convergence in the energy norm without stabilization in the symmetric and non-symmetric case. In the symmetric case we obtain additionally optimal convergence in the $L^{2}$-norm. In both cases the analysis relies on a discrete inf-sup condition drawing from earlier ideas on minimal stabilization for DG-methods in [6] and [15]. The analysis is also inspired by Larson and Niklasson [14] and extends some of their results to two and three space dimensions for first order polynomial approximations.

2. The problem setting. Let $\Omega$ be a convex polygon (polyhedron in three space dimensions) in $\mathbb{R}^{d}, d=2,3$, with outer normal $n$. Let $\mathcal{K}$ be a subdivision of $\Omega \subset \mathbb{R}^{d}$ into non-overlapping $d$-simplices $\kappa$. We consider the following elliptic problem with homogeneous Dirichlet boundary conditions:

Find $u: \Omega \rightarrow \mathbb{R}$ such that

$$
\left\{\begin{aligned}
-\nabla \cdot \sigma \nabla u & =f, \text { in } \Omega \\
u & =0, \text { on } \partial \Omega
\end{aligned}\right.
$$

where $f \in L^{2}(\Omega)$ and with diffusion coefficient $\left.\sigma(x)\right|_{\kappa}=\sigma_{\kappa} \in \mathbb{R}$ and $\sigma(x)>\sigma_{0}>0$. We assume that there exists a constant $\rho>0$ such that $\left.\sigma\right|_{\kappa_{1}} \leq\left.\rho \sigma\right|_{\kappa_{2}}$ for any two 
elements satisfying $\partial \kappa_{1} \cap \partial \kappa_{2} \neq \emptyset$. The fact that the boundary conditions are of homogeneous type is not a limitation of the presented methods but rather to avoid technical details.

Let $\mathcal{F}_{i}$ denote the set of interior faces $((d-1)$-manifolds) of the mesh, i.e. the set of faces that are not included in the boundary $\partial \Omega$. The set $\mathcal{F}_{e}$ denotes the faces that are included in $\partial \Omega$ and define $\mathcal{F}=\mathcal{F}_{i} \cup \mathcal{F}_{e}$. Note that for a subset $\mathcal{K}_{0} \subset \mathcal{K}$ we use the notation $\mathcal{F}_{i}\left(\mathcal{K}_{0}\right), \mathcal{F}_{e}\left(\mathcal{K}_{0}\right)$ to indicate the interior resp. exterior (with respect to $\mathcal{K}_{0}$ ) faces of the set $\mathcal{K}_{0}$.

Assume that $\mathcal{K}$ is shape-regular, does not contain any hanging node and covers $\bar{\Omega}$ exactly. Suppose that each $\kappa \in \mathcal{K}$ is an affine image of the reference element $\widehat{\kappa}$, i.e. for each element $\kappa$ there exists an affine transformation $T_{\kappa}: \widehat{\kappa} \rightarrow \kappa$. For an element $\kappa \in \mathcal{K}, h_{\kappa}$ denotes its diameter. Set $h=\max _{\kappa \in \mathcal{K}} h_{\kappa}$ and let $\tilde{h}, \tilde{m}$ be the functions such that $\left.\tilde{h}\right|_{\kappa}=h_{\kappa}$ resp. $\left.\tilde{m}\right|_{\kappa}=\operatorname{meas}(\kappa)$. We will say that a family of subdivisions $\{\mathcal{K}\}_{h}$ is asymptotically uniform with some $\zeta>0$ if there exists a constant $c>0$ such that for every $F \in \mathcal{F}_{i}$ there holds $|\tilde{m}|_{\kappa_{1}}-\left.\tilde{m}\right|_{\kappa_{2}}\left|\leq c h_{\kappa}^{\zeta} \tilde{m}\right|_{\kappa_{1}}$ where $F=\partial \kappa_{1} \cap \partial \kappa_{2}$ with $\kappa_{1}, \kappa_{2} \in\{\mathcal{K}\}_{h}$.

For a face $F \in \mathcal{F}, h_{F}$ denotes its diameter and let $\tilde{h}_{\mathcal{F}}$ be the function such that $\left.\tilde{h}_{\mathcal{F}}\right|_{F}=h_{F}$.

For a subset $R \subset \Omega$ or $R \subset \mathcal{F},(\cdot, \cdot)_{R}$ denotes the $L^{2}(R)$-scalar product, $\|\cdot\|_{R}=$ $(\cdot, \cdot)_{R}^{1 / 2}$ the corresponding norm, and $\|\cdot\|_{s, R}$ the $H^{s}(R)$-norm. The element-wise counterparts will be distinguished using the discrete partition as subscript, for example $(\cdot, \cdot)_{\mathcal{K}}=\sum_{\kappa \in \mathcal{K}}(\cdot, \cdot)_{K}$. For $s \geq 1$, let $H^{s}(\mathcal{K})$ be the space of piecewise Sobolev $H^{s_{-}}$ functions and denote its norm by $\|\cdot\|_{s, \mathcal{K}}$.

For $v \in H^{1}(\mathcal{K}), \tau \in\left[H^{1}(\mathcal{K})\right]^{d}$ and an interior face $F=\kappa_{1} \cap \kappa_{2} \in \mathcal{F}_{i}$, where $\kappa_{1}$ and $\kappa_{2}$ are two distinct elements of $\mathcal{K}$ with respective outer normals $n_{1}$ and $n_{2}$, define the jump and average by

$$
\begin{aligned}
{[v] } & =\left(\left.v\right|_{\kappa_{1}} n_{1}+\left.v\right|_{\kappa_{2}} n_{2}\right), & & \{v\}=\frac{1}{2}\left(\left.v\right|_{\kappa_{1}}+\left.v\right|_{\kappa_{2}}\right), \\
{[\tau] } & =\left(\left.\tau\right|_{\kappa_{1}} \cdot n_{1}+\left.\tau\right|_{\kappa_{2}} \cdot n_{2}\right), & & \{\tau\}=\frac{1}{2}\left(\left.\tau\right|_{\kappa_{1}}+\left.\tau\right|_{\kappa_{2}}\right) .
\end{aligned}
$$

Additionally we define on each face $F \in \mathcal{F}$ the normal $n_{F}$ in an arbitrary but fixed manner.

On outer faces $F=\partial \kappa \cap \partial \Omega \in \mathcal{F}_{e}$, for some $\kappa \in \mathcal{K}$ with outer normal $n$, the jump and the average are defined as $[v]=\left.v\right|_{F} n$ and $\{v\}=\left.v\right|_{F}$ resp. $[\tau]=\left.\tau\right|_{F} \cdot n$ and $\{\tau\}=\left.\tau\right|_{F}$.

The shape-regularity implies that there exists a constant $c>0$ independent of the mesh size $h$ such that on any face $F \in \mathcal{F}$

$$
h_{F} \leq\{\tilde{h}\} \leq c h_{F} .
$$

In this paper $c>0$ denotes a generic constant and can change at each occurrence, while an indexed constant stays fix. Any constant is independent of the mesh size $h$. We also introduce the average of the jump over a given face. Let $v \in H^{1}(\mathcal{K})$, then $\overline{[v]} \in \mathbb{R}^{d}$ is defined by

$$
\int_{F} \overline{[v]} d s=\int_{F}[v] d s \quad \forall F \in \mathcal{F} .
$$

3. Stabilization mechanisms for finite element methods. In this section we will recall some notions of the framework for stabilization mechanisms proposed in [5]. We first present the standard SIPG method and then we will introduce the 
modified methods that will be discussed in this paper. To this aim we introduce the broken space

$$
H_{\sigma}^{1}(\mathcal{K}):=\left\{\left.v\right|_{\kappa} \in H^{1}(\mathcal{K}) \text { such that }\left.\nabla \cdot(\sigma \nabla v)\right|_{\kappa} \in L^{2}(\kappa), \forall \kappa \in \mathcal{K}\right\} .
$$

A function $u \in H_{\sigma}^{1}(\mathcal{K})$ will coincide with a solution of (2.1) if and only if

$$
\begin{aligned}
\nabla \cdot(\sigma \nabla u) & =f \text { in each } \kappa \in \mathcal{K}, \\
{[u] } & =0 \text { on each } F \in \mathcal{F}, \\
{[\sigma \nabla u] } & =0 \text { on each } F \in \mathcal{F}_{i} .
\end{aligned}
$$

Following [5] we introduce a method in $H_{\sigma}^{1}(\mathcal{K})$ where these residuals are imposed to be zero weakly: Find $u \in H_{\sigma}^{1}(\mathcal{K})$ such that

$$
\left(\nabla \cdot(\sigma \nabla u)-f, B_{0} v\right)_{\mathcal{K}}+\left([u], \mathbf{B}_{1} v\right)_{\mathcal{F}}+\left([\sigma \nabla u], B_{2} v\right)_{\mathcal{F}_{i}}=0, \quad \forall v \in H_{\sigma}^{1}(\mathcal{K})
$$

where the three operators $B_{0}, \mathbf{B}_{1}$ and $B_{2}$ remain to be chosen and will define our method. The classical case of SIPG using penalization of the solution jumps is obtained by the choice

$$
\begin{aligned}
& B_{0} v=v \\
& \mathbf{B}_{1} v=-\{\sigma \nabla v\}+\gamma[v] \\
& B_{2} v=\{v\} .
\end{aligned}
$$

Inserting this in (3.1) and integrating by parts leads to the well known formulation: Find $u \in H_{\sigma}^{1}(\mathcal{K})$ such that

$$
(\sigma \nabla u, \nabla v)_{\mathcal{K}}-(\{\sigma \nabla u\},[v])_{\mathcal{F}}-(\{\sigma \nabla v\},[u])_{\mathcal{F}}+(\gamma[u],[v])_{\mathcal{F}}=(f, v)_{\mathcal{K}},
$$

for all $v \in H_{\sigma}^{1}(\mathcal{K})$. This formulation is symmetric and continuity is imposed by a penalization of the solution jump over element faces. The uniqueness of solutions to (3.2) is assured by Theorem 1 of [5] and the solution coincides with that of (2.1). In this paper we will investigate the possibility of choosing $\gamma=0$ on interior faces in (3.2). We show that the resulting discrete system always is well posed but that convergence depends on the regularity of the mesh and data. To relax the constraints on the data we then propose to work in a bubble enriched space.

For the modified methods proposed in this paper there is no coercivity and stability must be recovered using an inf-sup argument. This may seem unnatural for a method approximating an elliptic problem, but shows that when relaxing the stabilization the domain decomposition aspect of the DG-method is accentuated.

4. Finite Element Spaces. We will consider two low order finite element spaces in the present work, the space of piecewise affine discontinuous functions and the space of piecewise affine discontinuous functions enriched with nonconforming quadratic bubbles. We show that every function in the former space can be written as a sum of a midpoint continuous function (in the Crouzeix-Raviart space) and a "midpoint discontinuous" function in a space that will be specified later. The motivation for this decomposition is that the symmetric DG-bilinear form of (3.2) results in a block diagonal matrix for this choice of basis and hence the continuous and the discontinuous contributions may be analysed separately.

Define the piecewise affine discontinuous finite element space by

$$
V_{h}^{1}=\left\{v_{h} \in L^{2}(\Omega):\left.v_{h}\right|_{\kappa} \in \mathbb{P}_{1}(\kappa), \forall \kappa \in \mathcal{K}\right\}
$$


and the enriched space by

$$
V_{h}^{b}=V_{h}^{1} \oplus V^{b}
$$

with

$$
V^{b}=\left\{v \in L^{2}(\Omega):\left.v(x)\right|_{\kappa}=\alpha_{\kappa} x \cdot x ; \text { where } \alpha_{\kappa} \in \mathbb{R}\right\}
$$

where $x=\left(x_{1}, \ldots, x_{d}\right)$ denotes the physical variables. Additionally we define

$$
V_{h, 0}^{1}=\left\{v_{h} \in V_{h}^{1}: \int_{F} v_{h} d s=0, \forall F \in \mathcal{F}_{e}\right\}
$$

the space of piecewise affine elements where the homogeneous Dirichlet boundary conditions are imposed on the midpoints of each exterior face.

4.1. Splitting of the finite element space $V_{h, 0}^{1}$. The idea is to split $V_{h, 0}^{1}$ into a midpoint continuous space, the Crouzeix-Raviart space, and a midpoint discontinuous space. Recall the definition of the Crouzeix-Raviart space

$$
V^{C}=\left\{v_{h} \in V_{h}^{1}: \int_{F}\left[v_{h}\right] d s=0, \forall F \in \mathcal{F}_{i} ; \int_{F} v_{h} d s=0, \forall F \in \mathcal{F}_{e}\right\} .
$$

Its "midpoint discontinuous" counterpart is defined by

$$
V^{D}=\left\{v_{h} \in V_{h}^{1}: \int_{F}\left\{v_{h}\right\} d s=0, \forall F \in \mathcal{F}\right\} .
$$

Denote by $N_{i}$ the number of interior faces of the mesh $\mathcal{K}$. Let us denote $\left\{\phi_{i}^{c}\right\}_{i=1}^{N_{i}}$ the Crouzeix-Raviart basis defined such that

$$
\int_{F_{j}}\left\{\phi_{i}^{c}\right\} d s=\delta_{i, j} \operatorname{meas}\left(F_{i}\right), \quad \forall F_{j} \in \mathcal{F} .
$$

This builds a basis for the space $V^{C}$. Now let us define a basis for the space $V^{d}$. For each face $F$ consider the basis function $\phi_{F}^{d}$ defined as

$$
\left.\left(\phi_{F}^{d}\right)\right|_{\kappa}=\left.\frac{1}{2}\left(\phi_{F}^{c}\right)\right|_{\kappa} \frac{\left.\left(\nabla \phi_{F}^{c}\right)\right|_{\kappa} \cdot n_{F}}{\left|\left(\nabla \phi_{F}^{c}\right)\right|_{\kappa} \cdot n_{F} \mid}
$$

with $n_{F}$ a fixed but arbitrary normal associated to face $F$. It is easy to verify that $\phi_{i}^{d} \in V^{D}$ and that

$$
\int_{F_{j}}\left[\phi_{i}^{d}\right] \cdot n_{F_{j}} d s=\delta_{i, j} \operatorname{meas}\left(F_{i}\right), \quad \forall F_{i} \in \mathcal{F} .
$$

Now we are ready to prove the following lemma.

LEMMA 4.1. The splitting of $V_{h, 0}^{1}$ into $V^{C}$ and $V^{D}$ is a direct sum, i.e. $V_{h, 0}^{1}=$ $V^{C} \oplus V^{D}$. In addition any function $v_{h}$ in $V_{h, 0}^{1}$ can be written as

$$
v_{h}(x)=\sum_{i=1}^{N_{i}} c_{i} \phi_{i}^{c}(x)+\sum_{i=1}^{N_{i}} d_{i} \phi_{i}^{d}(x)
$$

where $c_{i}=\frac{1}{\operatorname{meas}\left(F_{i}\right)} \int_{F_{i}}\left\{v_{h}\right\} d s$ and $d_{i}=\frac{1}{\operatorname{meas}\left(F_{i}\right)} \int_{F_{i}}\left[v_{h}\right] \cdot n_{F_{i}} d s$. 
REMARK 4.2. Note that for $v \in H^{1}(\Omega)$ the function

$$
i_{c} v(x)=\sum_{i=1}^{N_{i}} c_{i} \phi_{i}^{c}(x)
$$

with $c_{i}=\frac{1}{m e a s\left(F_{i}\right)} \int_{F_{i}} v d s$ is the Crouzeix-Raviart interpolant and has optimal approximation properties.

Proof. First assume that $v_{h} \in V^{C} \cap V^{D}$ and show that $v_{h} \equiv 0$. Denote by $x_{F}$ the midpoint associated to the face $F \in \mathcal{F}$. Take an arbitrary exterior face $F$ and since $v_{h} \in V^{D}$

$$
0=\int_{F}\left\{v_{h}\right\} d s=\int_{F} v_{h} d s=\operatorname{meas}(F) v_{h}\left(x_{F}\right),
$$

using the midpoint integration rule which is exact for polynomials of order one. On the other hand for an interior face $F \in \mathcal{F}_{i}$ such that $F=\partial \kappa_{1} \cap \partial \kappa_{2}$ and since $v_{h} \in V^{D}$, we can write

$$
0=\int_{F}\left\{v_{h}\right\} d s=\frac{\operatorname{meas}(F)}{2}\left(\left.v_{h}\right|_{\kappa_{1}}\left(x_{F}\right)+\left.v_{h}\right|_{\kappa_{2}}\left(x_{F}\right)\right),
$$

and since $v_{h} \in V^{C}$

$$
0=\int_{F}\left[v_{h}\right] \cdot n_{F} d s=\operatorname{meas}(F)\left(\left.v_{h}\right|_{\kappa_{1}}\left(x_{F}\right) n_{1}+\left.v_{h}\right|_{\kappa_{2}}\left(x_{F}\right) n_{2}\right) \cdot n_{F} .
$$

Independent of the choice $n_{F}=n_{1}$ or $n_{F}=n_{2}$ we get

$$
\left.v_{h}\right|_{\kappa_{1}}\left(x_{F}\right)=\left.v_{h}\right|_{\kappa_{2}}\left(x_{F}\right)=0 \quad \forall F \in \mathcal{F}_{i} .
$$

Considering an arbitrary element $\kappa \in \mathcal{K}$ and respecting (4.2) and (4.3), observe that $v_{h}$ vanishes in the $d+1$ midpoints of the adjacent faces. Thus the function $v_{h} \in V_{h, 0}^{1}$ must be identically zero on all elements.

We show that $V^{C} \oplus V^{D}$ covers $V_{h, 0}^{1}$ entirely. Assume that $v_{h} \in V_{h, 0}^{1}$ and define $u_{h} \in V^{C} \oplus V^{D}$ by

$$
u_{h}(x)=\sum_{i=1}^{N_{i}}\left(c_{i} \phi_{i}^{c}(x)+d_{i} \phi_{i}^{d}(x)\right)
$$

with $c_{i}=\frac{1}{\operatorname{meas}\left(F_{i}\right)} \int_{F_{i}}\left\{v_{h}\right\} d s$ and $d_{i}=\frac{1}{\operatorname{meas}\left(F_{i}\right)} \int_{F_{i}}\left[v_{h}\right] d s$. Let us prove that $u_{h} \equiv v_{h}$. Integrating $u_{h}$ over an exterior face $F \in \mathcal{F}_{e}$ yields

$$
\int_{F} u_{h} d s=0=\int_{F} v_{h} d s
$$

Using again the midpoint integration rule implies

$$
u_{h}\left(x_{F}\right)=v_{h}\left(x_{F}\right) .
$$

On interior faces $F \in \mathcal{F}_{i}$ observe that

$$
\begin{gathered}
\int_{F}\left\{u_{h}\right\} d s=c_{F} \int_{F}\left\{\phi_{F}^{c}(x)\right\} d s=\operatorname{meas}(F) c_{F}=\int_{F}\left\{v_{h}\right\} d s, \\
\int_{F}\left[u_{h}\right] \cdot n_{F} d s=d_{F} \int_{F}\left[\phi_{F}^{d}(x)\right] \cdot n_{F} d s=\operatorname{meas}(F) d_{F}=\int_{F}\left[v_{h}\right] \cdot n_{F} d s,
\end{gathered}
$$


and thus

$$
\left.u_{h}\right|_{\kappa_{1}}\left(x_{F}\right)=\left.v_{h}\right|_{\kappa_{1}}\left(x_{F}\right) \text { and }\left.u_{h}\right|_{\kappa_{2}}\left(x_{F}\right)=\left.v_{h}\right|_{\kappa_{2}}\left(x_{F}\right) \text {. }
$$

Now since $v_{h}, u_{h} \in V_{h, 0}^{1}$ and since these two functions coincides at $d+1$ points per element they are equal. By consequence every function $v_{h}$ in $V_{h, 0}^{1}$ can be written as $v_{h}=v_{c}+v_{d}$ with $v_{c} \in V^{C}$ and $v_{d} \in V^{D}$.

LEMMA 4.3 (Asymptotic $L^{2}$-orthogonality between $V^{C}$ and $V^{D}$ ). Assume that the mesh is asymptotically uniform with some $\zeta>0$, then the spaces $V^{C}$ and $V^{D}$ satisfy the following weak $L^{2}$-orthogonality property: there exists a constant $c>0$ independent of $h$, such that

$$
\left|\left(v_{c}, v_{d}\right)_{\Omega}\right| \leq c h^{\zeta}\left\|v_{c}\right\|_{\Omega}\left\|v_{d}\right\|_{\Omega}+c\left\|\tilde{h} \nabla v_{c}\right\|_{\Omega}^{\star}\left\|\tilde{h} \nabla v_{d}\right\|_{\Omega}^{\star} \quad \forall v_{c} \in V^{C}, v_{d} \in V^{D},
$$

where the norms marked by $\star$ appear only in the case of $d=3$.

Proof. In the 2-dimensional case we can proceed as follows. Take $v_{c} \in V^{C}$ and $v_{d} \in V^{D}$ and develop

$$
\left|\left(v_{c}, v_{d}\right)_{\mathcal{K}}\right|=\left|\sum_{\kappa \in \mathcal{K}}\left(v_{c}, v_{d}\right)_{\kappa}\right|=\frac{1}{d+1}\left|\sum_{\kappa \in \mathcal{K}} \sum_{F \in \mathcal{F}(\kappa)} \tilde{m} v_{c}\left(x_{F}\right) v_{d}\left(x_{F}\right)\right|
$$

using the midpoint integration rule which integrates over the volume $\kappa$ in function of the integrand evaluated in the midpoints of each face. This integration rule is exact of order 2 in the 2-dimensional case but not in the 3 -dimensional one. The points $x_{F}$ denote the midpoints of the face $F$. Since $v_{d}\left(x_{F}\right)=0$ for exterior faces $F \in \mathcal{F}_{e}$ and since $v_{c}$ is midpoint continuous we can rearrange the sum

$$
\left|\left(v_{c}, v_{d}\right)_{\mathcal{K}}\right|=\frac{2}{d+1}\left|\sum_{F \in \mathcal{F}_{i}} v_{c}\left(x_{F}\right)\left\{\tilde{m} v_{d}\right\}\left(x_{F}\right)\right| .
$$

Since $\{u v\}=\{u\}\{v\}+\frac{1}{4}[u] \cdot[v]$ and $\left\{v_{d}\right\}\left(x_{F}\right)=0$ for all interior faces

$$
\left|\left(v_{c}, v_{d}\right)_{\mathcal{K}}\right| \leq \frac{2}{d+1} \sum_{F \in \mathcal{F}_{i}}\left|v_{c}\left(x_{F}\right)\right|\left|[\tilde{m}]\left(x_{F}\right) \cdot n_{F}\right|\left|\left[v_{d}\right]\left(x_{F}\right) \cdot n_{F}\right| .
$$

The regularity assumption on the mesh implies that $\left|[\tilde{m}] \cdot n_{F}\right| \leq \operatorname{ch}^{\zeta}\{\tilde{m}\}$ with $\zeta>0$ and since $\frac{1}{2}\left|\left[u_{d}\right]\left(x_{F}\right) \cdot n_{F}\right|=\left|u_{d}\right|_{\kappa_{i}} \mid$, for $i=1,2$, we can rearrange the sum again

$$
\left|\left(v_{c}, v_{d}\right)_{\mathcal{K}}\right| \leq c h^{\zeta} \sum_{\kappa \in \mathcal{K}}\left(\left|v_{c}\right|,\left|v_{d}\right|\right)_{\kappa} \leq c h^{\zeta}\left\|v_{c}\right\|_{\Omega}\left\|v_{d}\right\|_{\Omega} .
$$

In the 3-dimensional case, since the midpoint integration rule is not anymore of order two, we introduce the local midpoint interpolation $i_{h}^{\kappa}$ for each element. Then using the triangle inequality yields

$$
\left|\left(v_{c}, v_{d}\right)_{\mathcal{K}}\right| \leq\left|\sum_{\kappa \in \mathcal{K}} \int_{\kappa} i_{h}^{\kappa}\left(v_{c} v_{d}\right) d x\right|+\left|\sum_{\kappa \in \mathcal{K}} \int_{\kappa}\left(v_{c} v_{d}-i_{h}^{\kappa}\left(v_{c} v_{d}\right)\right) d x\right| .
$$

The first term of the right hand side of (4.5) can be developed as in (4.4) since now for the midpoint interpolation $i_{h}^{\kappa}$ the midpoint integration rule is exact. For the second term of the right hand side of (4.5) one can show using standard interpolation results that

$$
\left|\sum_{\kappa \in \mathcal{K}} \int_{\kappa}\left(v_{c} v_{d}-i_{h}^{\kappa}\left(v_{c} v_{d}\right)\right) d x\right| \leq c\left\|\tilde{h} \nabla v_{c}\right\|\left\|_{\mathcal{K}}\right\| \tilde{h} \nabla v_{d} \|_{\mathcal{K}}
$$

$\square$ 
4.2. Properties of the enriched space $V_{h}^{b}$. The motivation for the particular form of the enriched space is given in the following Lemma. The key idea is that the gradient of a function in $V_{h}^{b}$ restricted to an element is in fact locally in the RaviartThomas space and equivalently the curl of a function in $\left[V_{h}^{b}\right]^{d}$ is locally in the lowest order Nedelec space. Here we will only exploit the former property. Let $R T_{0}$ denote the space of Raviart-Thomas elements of order zero.

Lemma 4.4. For all $w_{h} \in V_{h}^{b}$ there holds

$$
\left.\nabla w_{h}\right|_{\kappa} \in R T_{0}(\kappa),
$$

and for all $r_{h} \in R T_{0}(\kappa)$ there exists $w_{h} \in V_{h}^{b}$ such that $\left.\nabla w_{h}\right|_{\kappa}=r_{h}$, for all $\kappa \in \mathcal{K}$.

Proof. Let $w_{h} \in V_{h}^{b}$, restricting $w_{h}$ to an arbitrary element $\kappa$ we can write

$$
\left.w_{h}\right|_{\kappa}(x)=\alpha x \cdot x+\beta \cdot x+\gamma
$$

where $\alpha, \gamma \in \mathbb{R}$ and $\beta \in \mathbb{R}^{d}$ are the local degrees of freedom. Then

$$
\left.\nabla w_{h}\right|_{\kappa}(x)=2 \alpha x+\beta .
$$

To show that this function lies in the Raviart-Thomas finite element space we have to map it on the reference element using the Piola transformation. But let us first introduce the affine transformation $T_{\kappa}$ between the reference element $\widehat{\kappa}$ defined by its vertices $a_{i}=e_{i}$ for $i=1, . ., d$ and $a_{d+1}=\mathcal{O}$ and the physical element $\kappa$. The vectors $e_{i}$ denote the unit vectors corresponding to the $i$-th coordinate. The affine transformation may be written as

$$
T_{\kappa}(\hat{x})=J_{\kappa} \hat{x}+t_{\kappa}
$$

where $\hat{x}=\left(\hat{x}_{1}, \ldots, \hat{x}_{d}\right)^{\top} \in \widehat{\kappa}$ denotes the variable in the reference element. Then we denote by $\psi_{\kappa}$ the Piola transformation between the physical element and the reference element defined by

$$
\psi_{\kappa}(v)(\hat{x})=\left|J_{\kappa}\right| J_{\kappa}^{-1} v\left(T_{\kappa}(\hat{x})\right)
$$

Thus

$$
\psi_{\kappa}\left(\left.\nabla w_{h}\right|_{\kappa}\right)(\hat{x})=\left|J_{\kappa}\right| J_{\kappa}^{-1}\left(2 \alpha T_{\kappa}(\hat{x})+\beta\right)=\left|J_{\kappa}\right|\left(2 \alpha \hat{x}+J_{\kappa}^{-1}\left(\beta+2 \alpha t_{\kappa}\right)\right)
$$

and this function is clearly an element of the Raviart-Thomas finite element space on the reference element.

On the other hand if $r_{h} \in R T_{0}$ then $\left.\psi_{\kappa} \circ r_{h}\right|_{\kappa}$ is of the form

$$
\psi_{\kappa}\left(\left.r_{h}\right|_{\kappa}\right)(\hat{x})=a \hat{x}+b
$$

where $a \in \mathbb{R}$, and $b=\left(b_{1}, \ldots, b_{d}\right)^{\top} \in \mathbb{R}^{d}$. Thus

$$
\left.r_{h}\right|_{\kappa}(x)=\frac{1}{\left|J_{\kappa}\right|}\left(a x+J_{\kappa} b-t_{\kappa}\right) .
$$

Defining locally

$$
\left.w_{h}\right|_{\kappa}(x)=\frac{1}{\left|J_{\kappa}\right|}\left(\frac{a}{2} x \cdot x+\left(J_{\kappa} b-t_{\kappa}\right) \cdot x\right)
$$

yields that

$$
\left.\nabla w_{h}\right|_{\kappa}(x)=\left.r_{h}\right|_{\kappa}(x) .
$$

(1) 
5. Poincaré Inequalities. The analysis of the model problem (2.1) relies on the Poincaré inequality. It is therefore important to show that Poincaré inequalities hold for the different spaces used. First we give an elementary result showing that only the average solution jump needs to be controlled for a Poincaré inequality to hold in a DG-method. Then we prove Poincare inequalities for the two spaces $V^{C}$ and $V^{D}$ separately, a result that is of essential importance to prove the well posedness of the symmetric unstabilized formulation. Finally we prove a stronger Poincaré inequality for the space $V^{D}$ under some mesh-assumption in order to prove optimal convergence of the unstabilized formulation.

LEMma 5.1. There is a constant $c>0$ independent of $h$ such that for all $u_{h} \in V_{h}^{b}$ there holds

$$
c\left\|\omega^{\frac{1}{2}} \tilde{h}_{\mathcal{F}}^{-\frac{1}{2}}\left[u_{h}\right]\right\|_{\mathcal{F}}^{2} \leq\left\|\omega^{\frac{1}{2}} \tilde{h}_{\mathcal{F}}^{-\frac{1}{2}} \overline{\left[u_{h}\right]}\right\|_{\mathcal{F}}^{2}+\left\|\sigma^{\frac{1}{2}} \nabla u_{h}\right\|_{\mathcal{K}}^{2}
$$

Proof. Immediate by the approximation properties of the average jump and a discrete trace inequality:

$$
\begin{aligned}
\left\|\omega^{\frac{1}{2}} \tilde{h}_{\mathcal{F}}^{-\frac{1}{2}}\left[u_{h}\right]\right\|_{\mathcal{F}}^{2} & =\left\|\omega^{\frac{1}{2}} \tilde{h}_{\mathcal{F}}^{-\frac{1}{2}} \overline{\left[u_{h}\right]}\right\|_{\mathcal{F}}^{2}+\left\|\omega^{\frac{1}{2}} \tilde{h}_{\mathcal{F}}^{-\frac{1}{2}}\left(\left[u_{h}\right]-\overline{\left[u_{h}\right]}\right)\right\|_{\mathcal{F}}^{2} \\
& \leq\left\|\omega^{\frac{1}{2}} \tilde{h}_{\mathcal{F}}^{-\frac{1}{2}} \overline{\left[u_{h}\right]}\right\|_{\mathcal{F}}^{2}+c\left\|\omega^{\frac{1}{2}} \tilde{h}_{\mathcal{F}}^{\frac{1}{2}}\left[\nabla u_{h} \times n\right]\right\|_{\mathcal{F}}^{2} \\
& \leq\left\|\omega^{\frac{1}{2}} \tilde{h}_{\mathcal{F}}^{-\frac{1}{2}} \overline{\left[u_{h}\right]}\right\|_{\mathcal{F}}^{2}+c\left\|\sigma^{\frac{1}{2}} \nabla u_{h}\right\|_{\mathcal{K}}^{2} .
\end{aligned}
$$

Corollary 5.2. The following Poincaré inequality for broken $H^{1}$-spaces holds, for all $u_{h} \in V_{h}^{b}$

$$
c\left\|\sigma^{\frac{1}{2}} u_{h}\right\|_{\mathcal{K}}^{2} \leq\left\|\omega^{\frac{1}{2}} \tilde{h}_{\mathcal{F}}^{-\frac{1}{2}} \overline{\left[u_{h}\right]}\right\|_{\mathcal{F}}^{2}+\left\|\sigma^{\frac{1}{2}} \nabla u_{h}\right\|_{\mathcal{K}}^{2}
$$

Proof. An immediate consequence of the previous lemma and the Poincaré inequality

$$
c\left\|\sigma^{\frac{1}{2}} u_{h}\right\|_{\mathcal{K}}^{2} \leq\left\|\omega^{\frac{1}{2}} \tilde{h}_{\mathcal{F}}^{-\frac{1}{2}}\left[u_{h}\right]\right\|_{\mathcal{F}}^{2}+\left\|\sigma^{\frac{1}{2}} \nabla u_{h}\right\|_{\mathcal{K}}^{2}
$$

proved by Brenner [4].

REMARK 5.3. We give the proofs for $u_{h} \in V_{h}^{b}$ but since $V_{h}^{1} \subset V_{h}^{b}$ the above results hold also for the standard space of piecewise affine discontinuous functions.

Proposition 5.4 (Poincaré inequality for $V^{C}$ ). There exists a constant $c>0$ depending only on $\Omega$ such that, for all $h<1$,

$$
\forall u_{c} \in V^{C}, \quad c\left\|u_{c}\right\|_{\mathcal{K}} \leq\left|u_{c}\right|_{1, \mathcal{K}} .
$$

Proof. See Temam [21] or Ern-Guermond [11] ם

Proposition 5.5 (Poincaré inequality for $V^{D}$ ). There exists a constant $c>0$ depending only on $\Omega$ such that, for all $h<1$,

$$
\forall u_{d} \in V^{D}, \quad c\left\|u_{d}\right\|_{\mathcal{K}} \leq\left|u_{d}\right|_{1, \mathcal{K}} .
$$




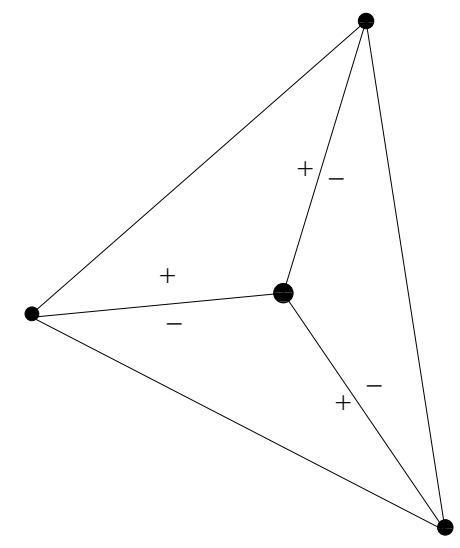

FIG. 5.1. Illustration of the macroelement argument of Lemma 5.6 the ' + ' and '-' signs refer to the sign of $u_{d}$ at the midpoints of the faces

Proof. Let $u_{d} \in V^{D}$ be fixed. Then define the splitting of $\Omega$ into two parts $\mathcal{K}_{1}$ and $\mathcal{K}_{2}$ by

$$
\begin{aligned}
& \mathcal{K}_{1}=\left\{\kappa \in \mathcal{K}: \exists x \in \kappa \text { s.t. } u_{d}(x)=0\right\} \\
& \mathcal{K}_{2}=\left\{\kappa \in \mathcal{K}: u_{d}(x) \neq 0, \forall x \in \kappa\right\} .
\end{aligned}
$$

Firstly prove the inequality for the region $\mathcal{K}_{1}$. Fix an element $\kappa_{1} \in \mathcal{K}_{1}$ and define

$$
Z\left(\kappa_{1}\right)=\left\{x \in \kappa_{1}: u_{d}(x)=0\right\} .
$$

Observe that $Z\left(\kappa_{1}\right)$ is either $\kappa_{1}$ itself, a line or one of the vertices since $\left.u_{d}\right|_{\kappa_{1}} \in \mathbb{P}_{1}\left(\kappa_{1}\right)$. However we may write in all three cases

$$
u_{d}(x)=\nabla u_{d} \cdot\left(x-x^{\star}\right) \quad \text { with } x^{\star} \in Z\left(\kappa_{1}\right) .
$$

Thus we conclude immediately that

$$
\left\|u_{d}\right\|_{\mathcal{K}_{1}} \leq\left\|\tilde{h} \nabla u_{d}\right\|_{\mathcal{K}_{1}} .
$$

Secondly split $\mathcal{K}_{2}$ in maximal subsets $\left\{\mathcal{K}_{2}^{j}\right\}_{j=1}^{m}$ in order that each

$$
\Omega_{2}^{j}=\left(\bigcup_{\kappa \in \mathcal{K}_{2}^{j}}^{\circ} \kappa\right)
$$

is connex. Fix a subset $\mathcal{K}_{2}^{j}$ and observe that $\left|u_{d}\right|$ is midpoint continuous on interior faces of $\mathcal{K}_{2}^{j}$. In consequence $\left|u_{d}\right|$ lies in the Crouzeix-Raviart space over the domain $\Omega_{2}^{j}$ and thus let us proceed analogous to the proof of Proposition 5.4. Details of the remaining part are given in the appendix.

In case the mesh has a certain macro-element structure we may prove a stronger Poincaré inequality for the space $V^{D}$.

Proposition 5.6 (Strong Poincaré inequality for $V^{D}$ ). Let $\mathcal{K}$ be a mesh. Assume that there exists a coarse mesh $\mathcal{T}$ covering $\bar{\Omega}$ such that each macro element (d-simplex) 
$T \in \mathcal{T}$ contains exactly $d+1$ elements $\kappa_{1}, \ldots, \kappa_{d+1}$ of $\mathcal{K}$ and such that $\kappa_{i} \cap \kappa_{j} \neq \emptyset$, for all $1 \leq i, j \leq d+1$. Then the following inequality holds

$$
\forall u_{d} \in V^{D}, \quad c\left\|u_{d}\right\|_{\mathcal{K}} \leq\left|\tilde{h} u_{d}\right|_{1, \mathcal{K}} .
$$

Proof. Let $u_{d} \in V^{D}$ and fix an element $\kappa \in \mathcal{K}$. If there exists $x^{\star} \in \kappa$ such that $u_{d}\left(x^{\star}\right)=0$ we conclude analogous to $(5.2)$ that

$$
\left\|u_{d}\right\|_{\kappa} \leq\left\|h_{\kappa} \nabla u_{d}\right\|_{\kappa} .
$$

Otherwise there exists in the same macro element $T \in \mathcal{T}$ a neighbour element $\kappa^{\prime} \in \mathcal{K}$ such that there exists $x^{\star} \in \kappa^{\prime}$ with $u_{d}\left(x^{\star}\right)=0$. Indeed assume that $u_{d}(x) \neq 0$ for all $x \in T$. Observe that $u_{d}$ changes sign in the midpoint of each face since it lies in $V^{D}$ and hence $\int_{F}\left\{u_{d}\right\}=0$. Consider all three elements of $T$ in 2 dimensions and an arbitrary selection of three elements in 3 dimensions. The solution changes sign over each face. However in the three elements the sign has to change four times. Hence it has to change sign within one element which leads to a contradiction. See the illustration in Figure 5.1. Thus there exists at least one element $\kappa^{\star} \in \mathcal{K}$ of the macro element $T$ such that there exists a point $x^{\star} \in \kappa^{\star}$ with $u_{d}\left(x^{\star}\right)=0$. Since $\kappa \cap \kappa^{\star} \neq 0$ we conclude that

$$
\left\|u_{d}\right\|_{\kappa} \leq c\left\|h_{\kappa} \nabla u_{d}\right\|_{\kappa}
$$

6. Discontinuous Galerkin Methods. Define the following bilinear form

$$
a_{s}\left(u_{h}, v_{h}\right)=\left(\sigma \nabla u_{h}, \nabla v_{h}\right)_{\mathcal{K}}-\left(\left\{\sigma \nabla u_{h}\right\},\left[v_{h}\right]\right)_{\mathcal{F}}-s\left(\left\{\sigma \nabla v_{h}\right\},\left[u_{h}\right]\right)_{\mathcal{F}},
$$

for $s \in\{-1,1\}$ and the stabilization term

$$
j\left(u_{h}, v_{h}\right)=\left(\omega \tilde{h}_{\mathcal{F}}^{-1} \overline{\left[u_{h}\right]}, \overline{\left[v_{h}\right]}\right)_{\mathcal{F}_{i}},
$$

where $\left.\omega\right|_{F}=\min \left(\left.\sigma\right|_{\kappa_{1}},\left.\sigma\right|_{\kappa_{2}}\right)$ for $F=\partial \kappa_{1} \cap \partial \kappa_{2}$. Note that we have replaced the penalization of the jumps of formulation (3.2) with the penalization of the average jumps in the spirit of Lemma 5.1.

Let us define two methods to approximate the solution of (2.1):

Method 1: Find $u_{h}^{1} \in V_{h, 0}^{1}$ such that

$$
a_{s}\left(u_{h}^{1}, v_{h}\right)+\gamma j\left(u_{h}^{1}, v_{h}\right)=\left(f, v_{h}\right)_{\mathcal{K}} \quad \forall v_{h} \in V_{h, 0}^{1},
$$

for some $\gamma \in \mathbb{R}$ and $s \in\{-1,1\}$.

REMARK 6.1. Except for the penalization of the average jump only this corresponds to the SIPG-method for $s=1$ and the NIPG-method for $s=-1$. The well-posedness of the particular case $\gamma=0$ is discussed in Lemma 8.6, the convergence behavior in Section §8.2.

Method 2: Find $u_{h}^{b} \in V_{h}^{b}$ such that

$$
a_{s}\left(u_{h}^{b}, v_{h}\right)=\left(f, v_{h}\right)_{\mathcal{K}} \quad \forall v_{h} \in V_{h}^{b} .
$$

REMARK 6.2 (Local mass conservation property). The solutions $u_{h}^{1}, u_{h}^{b}$ of (6.2) resp. (6.3) satisfy

$$
\begin{aligned}
-\int_{\partial \kappa}\left\{\sigma \nabla u_{h}^{1}\right\} \cdot n_{\kappa} d s+\gamma & \int_{\partial \kappa} \omega \tilde{h}_{\mathcal{F}}^{-1} \overline{\left[u_{h}^{1}\right]} \cdot n_{\kappa} d s=\int_{\kappa} f d x, \\
-\int_{\partial \kappa}\left\{\sigma \nabla u_{h}^{b}\right\} \cdot n_{\kappa} d s & =\int_{\kappa} f d x .
\end{aligned}
$$


Remark that $u_{h}^{b}$ is locally mass conservative while for $u_{h}^{1}$ with $\gamma \neq 0$ the local flux includes the penalty term depending on the stabilization parameter $\gamma$.

Lemma 6.3 (Consistency of methods). If the exact solution $u$ of problem (2.1) satisfies $u \in H^{1}(\Omega)$, then the formulations defined by (6.2) and (6.3) are consistent (and adjoint consistent if the bilinear form is symmetric). Moreover the following Galerkin orthogonalities hold

$$
\begin{aligned}
a_{s}\left(u-u_{h}^{1}, v_{h}\right)+\gamma j\left(u-u_{h}^{1}, v_{h}\right) & =0 & & \forall v_{h} \in V_{h, 0}^{1} \\
a_{s}\left(u-u_{h}^{b}, v_{h}\right) & =0 & & \forall v_{h} \in V_{h}^{b}
\end{aligned}
$$

where $u_{h}^{1} \in V_{h, 0}^{1}$ and $u_{h}^{b} \in V_{h}^{b}$ denote the discrete solutions of (6.2) resp. (6.3).

Proof. Since the form $a_{s}(\cdot, \cdot)$ coincides with the SIPG formulation for $s=1$ resp. NIPG for $s=-1$ it is consistent. Moreover for $s=1$ the method is adjoint consistent. We only show the primal consistency, dual consistency follows by symmetry. Since $u \in H^{1}(\Omega)$ we have

$$
\left.[u]\right|_{F}=0, \quad \forall F \in \mathcal{F}, \quad \forall F \in \mathcal{F}_{i}
$$

and by partial integration

$$
a_{s}\left(u, v_{h}\right)=\left(\sigma \nabla u, \nabla v_{h}\right)_{\mathcal{K}}-\left(\sigma \nabla u,\left[v_{h}\right]\right)_{\mathcal{F}}=\left(\nabla \cdot(\sigma \nabla u), v_{h}\right)_{\mathcal{K}}=\left(f, v_{h}\right)_{\mathcal{K}}
$$

for all $v_{h} \in V_{h}^{b}$. Note that $V_{h, 0}^{1} \subset V_{h}^{b}$. Observe also that for $u$, solution of (2.1), there holds

$$
j\left(u, v_{h}\right)=\left(\omega \tilde{h}_{\mathcal{F}}^{-1} \overline{[u]}, \overline{\left[v_{h}\right]}\right)_{\mathcal{F}_{i}}=0,
$$

which concludes the proof.

7. Technical results. For the analysis it is useful to introduce the following norms

$$
\begin{aligned}
\left\|v_{h}\right\|^{2} & =\left\|\sigma^{\frac{1}{2}} v_{h}\right\|_{1, \mathcal{K}}^{2}+\left\|\omega^{\frac{1}{2}} \tilde{h}_{\mathcal{F}}^{-\frac{1}{2}}\left[v_{h}\right]\right\|_{\mathcal{F}}^{2}, \\
\left\|v_{h}\right\|_{d}^{2} & =\left\|\sigma^{\frac{1}{2}} \nabla v_{h}\right\|_{\mathcal{K}}^{2}+\left\|\omega^{\frac{1}{2}} \tilde{h}_{\mathcal{F}}^{-\frac{1}{2}}\left[v_{h}\right]\right\|_{\mathcal{F}}^{2}, \\
\left\|v_{h}\right\|_{c}^{2} & =\left\|\sigma^{\frac{1}{2}} \nabla v_{h}\right\|_{\mathcal{K}}^{2}+\left\|\tilde{h}_{\mathcal{F}}^{\frac{1}{2}}\left\{\sigma \nabla v_{h}\right\}\right\|_{\mathcal{F}_{i}}^{2},
\end{aligned}
$$

for all $v_{h} \in V_{h}^{b}$. Using the Poincaré inequality (5.1) we have

$$
\left\|v_{h}\right\| \leq c\left\|v_{h}\right\|_{d} .
$$

We have the following standard approximability results.

Lemma 7.1 (Approximability in $V_{h}^{1}$ ). Let $u \in H^{2}(\Omega)$ and $\pi_{h} u \in V_{h}^{1}$ denote the element-wise $L^{2}$-projection of $u$ onto $V_{h}^{1}$, then there holds

$$
\|\| u-\pi_{h} u\|\mid \leq c h\| u \|_{2, \mathcal{K}} \quad \text { and } \quad\left\|u-\pi_{h} u\right\|_{c} \leq c h\|u\|_{2, \mathcal{K}} .
$$

Proof. The claim is an immediate consequence of the following inequalities

$$
\left\|u-\pi_{h} u\right\|_{\mathcal{K}} \leq c h^{2}\|u\|_{2, \mathcal{K}}, \quad\left\|\nabla\left(u-\pi_{h} u\right)\right\|_{\mathcal{K}} \leq c h\|u\|_{2, \mathcal{K}}
$$

and

$$
\left\|\tilde{h}_{\mathcal{F}}^{-\frac{1}{2}}\left[u-\pi_{h} u\right]\right\|_{\mathcal{F}} \leq c h\|u\|_{2, \mathcal{K}}, \quad\left\|\tilde{h}_{\mathcal{F}}^{\frac{1}{2}}\left\{\nabla\left(u-\pi_{h} u\right)\right\}\right\|_{\mathcal{F}} \leq c h\|u\|_{2, \mathcal{K}}
$$


that are proved using trace inequalities and standard approximation results. $\mathrm{C}$

LEMMA 7.2 (Approximability in $V^{C}$ ). Let $u \in H^{\beta}(\Omega)$, with $\beta \in\{1,2\}$, and $i_{c} u \in V^{C}$ denote the Crouzeix-Raviart-interpolant of $u$ onto $V^{C}$ defined by $(4.1)$, then there holds

$$
\left\|u-i_{c} u\right\|_{\mathcal{K}} \leq c h^{\beta}\|u\|_{\beta, \mathcal{K}}
$$

If $u \in H^{2}(\Omega)$, then

$$
\begin{array}{r}
\left\|u-i_{c} u\right\| \leq c h\|u\|_{2, \mathcal{K}}, \\
\left\|u-i_{c} u\right\|_{c} \leq c h\|u\|_{2, \mathcal{K}} .
\end{array}
$$

Proof. We refer to the original paper of Crouzeix and Raviart [9] for the optimal approximation properties of the interpolant.

\section{Analysis of method 1 .}

8.1. Stability. In this section we will use orthogonality properties of $V^{C}$ and $V^{D}$ to obtain coercivity results also for the unstabilized method. These results ensures existence of the discrete solution.

LEMmA 8.1 (Orthogonality relations). The bilinear forms $a_{s}(\cdot, \cdot)$ and $j(\cdot, \cdot)$ satisfy the following orthogonality relations:

$$
\begin{aligned}
a_{s}\left(v_{c}, v_{d}\right) & =0 & & \forall v_{c} \in V^{C}, \forall v_{d} \in V^{D}, \\
a_{s}\left(v_{d}, v_{c}\right) & =(1-s)\left(\sigma \nabla v_{d}, \nabla v_{c}\right)_{\mathcal{K}} & & \forall v_{c} \in V^{C}, \forall v_{d} \in V^{D}, \\
j\left(v_{c}, v_{d}\right) & =j\left(v_{d}, v_{c}\right)=0 & & \forall v_{c} \in V^{C}, \forall v_{d} \in V^{D} .
\end{aligned}
$$

REMARK 8.2. The spaces $V^{C}$ and $V^{D}$ are are orthogonal with respect to the symmetric bilinear form $a_{1}(\cdot, \cdot)$.

Proof. Let $v_{c} \in V^{C}$ and $v_{d} \in V^{D}$. Since $\int_{F}\left[v_{c}\right] d x=0$ for all interior faces $F \in \mathcal{F}_{i}$ it follows directly that

$$
j\left(v_{c}, v_{d}\right)=j\left(v_{d}, v_{c}\right)=0
$$

and that

$$
a_{s}\left(v_{c}, v_{d}\right)=\left(\sigma \nabla v_{c}, \nabla v_{d}\right)_{\mathcal{K}}-\left(\left\{\sigma \nabla v_{c}\right\},\left[v_{d}\right]\right)_{\mathcal{F}_{i}}
$$

An integration by parts yields

$$
a_{s}\left(v_{c}, v_{d}\right)=-\left(\nabla \cdot \sigma \nabla v_{c}, v_{d}\right)_{\mathcal{K}}+\left(\left[\sigma \nabla v_{c}\right],\left\{v_{d}\right\}\right)_{\mathcal{F}}=0,
$$

since $\int_{F}\left\{v_{d}\right\} d x=0$ for all faces $F \in \mathcal{F}$ and $\nabla \cdot \sigma \nabla v_{c} \equiv 0$. Analogously we prove that

$$
a_{s}\left(v_{d}, v_{c}\right)=\left(\sigma \nabla v_{d}, \nabla v_{c}\right)_{\mathcal{K}}-s\left(\left\{\sigma \nabla v_{c}\right\},\left[v_{d}\right]\right)_{\mathcal{F}_{i}}=(1-s)\left(\sigma \nabla v_{d}, \nabla v_{c}\right)_{\mathcal{K}}
$$

LEMma 8.3. The bilinear forms $a_{s}(\cdot, \cdot)$ and $j(\cdot, \cdot)$ satisfy the following relations:

$$
\begin{aligned}
a_{s}\left(u_{c}, v_{c}\right) & =\left(\sigma \nabla u_{c}, \nabla v_{c}\right)_{\mathcal{K}} & & \forall u_{c}, v_{c} \in V^{C}, \\
a_{s}\left(u_{d}, v_{d}\right) & =-s\left(\sigma \nabla u_{d}, \nabla v_{d}\right)_{\mathcal{K}} & & \forall u_{d}, v_{d} \in V^{D}, \\
j\left(u_{c}, v_{c}\right) & =0 & & \forall u_{c}, v_{c} \in V^{C} .
\end{aligned}
$$


Proof. The proof is similar to the one of Lemma 8.1 and uses the properties of the spaces $V^{C}$ and $V^{D}$.

LEMMA 8.4 (Splitting of method 1). The first method defined by (6.2) is equivalent to: Find $u_{c} \in V^{C}, u_{d} \in V^{D}$ such that

$$
\begin{aligned}
\left(\sigma \nabla u_{c}, \nabla v_{c}\right)_{\mathcal{K}}+(1-s)\left(\sigma \nabla u_{d}, \nabla v_{c}\right)_{\mathcal{K}}=\left(f, v_{c}\right)_{\mathcal{K}} & \forall v_{c} \in V^{C} \\
-s\left(\sigma \nabla u_{d}, \nabla v_{d}\right)_{\mathcal{K}}+\gamma\left(\omega \tilde{h}_{\mathcal{F}}^{-1} \overline{\left[u_{d}\right]}, \overline{\left[v_{d}\right]}\right)_{\mathcal{F}_{i}}=\left(f, v_{d}\right)_{\mathcal{K}} & \forall v_{d} \in V^{D} .
\end{aligned}
$$

REMARK 8.5. Observe that for $s=1$, (8.1) is the Crouzeix-Raviart method for problem (2.1). In consequence the stability and convergence analysis is known for this problem. Since in this case $u_{c}$ is totally uncoupled of the second problem $(8.2), u_{c}$ always converges to the exact solution with optimal rate independently of the choice of the stabilization parameter, $\gamma=0$ included. Moreover note that (8.2) is independent of (8.1). Hence we can solve first for the discontinuous field $u_{d}$ and the for the continuous field $u_{c}$ also in the case $s=-1$.

Proof. Let $v_{h} \in V_{h, 0}^{1}$. Since $V_{h, 0}^{1}=V^{C} \oplus V^{D}$ we can write $v_{h}=v_{c}+v_{d}$ with $v_{c} \in V^{C}$ and $v_{d} \in V^{D}$. Analogously we can write $u_{h}^{1}=u_{c}+u_{d}$. Testing in (6.2) with $v_{c}$ and $v_{d}$ separately yields the problem: Find $u_{c} \in V^{C}$ and $u_{d} \in V^{D}$ such that

$$
\begin{array}{ll}
a_{s}\left(u_{c}+u_{d}, v_{c}\right)+\gamma j\left(u_{c}+u_{d}, v_{c}\right)=\left(f, v_{c}\right)_{\mathcal{K}} & \forall v_{c} \in V^{C} \\
a_{s}\left(u_{c}+u_{d}, v_{d}\right)+\gamma j\left(u_{c}+u_{d}, v_{d}\right)=\left(f, v_{d}\right)_{\mathcal{K}} & \forall v_{d} \in V^{D} .
\end{array}
$$

Applying Lemma 8.1 leads directly to: Find $u_{c} \in V^{C}$ and $u_{d} \in V^{D}$ such that

$$
\begin{aligned}
a_{s}\left(u_{c}+u_{d}, v_{c}\right)=\left(f, v_{c}\right)_{\mathcal{K}} & \forall v_{c} \in V^{C} \\
a_{s}\left(u_{d}, v_{d}\right)+\gamma j\left(u_{d}, v_{d}\right)=\left(f, v_{d}\right)_{\mathcal{K}} & \forall v_{d} \in V^{D} .
\end{aligned}
$$

Note that the equivalences between the problems (8.3) and (8.1) resp. (8.4) and (8.2) follow directly from Lemma 8.3.

LEMMA 8.6 (Coercivity of method 1). The discrete problem (8.2) is well posed for all $s \gamma \leq 0$ and $s \gamma>C_{\text {stab }}$, where $C_{\text {stab }}>0$ is a certain constant independent on h, i.e.

$$
C(\gamma)\left|\left\|u_{d}\right\|_{d}^{2} \leq\right| a_{s}\left(u_{d}, u_{d}\right)+\gamma j\left(u_{d}, u_{d}\right) \mid
$$

where

$$
C(\gamma)= \begin{cases}c \min (1,|\gamma|) & \text { if } s \gamma<0, \\ c\left(\gamma-C_{\text {stab }}\right) & \text { if } s \gamma>C_{\text {stab }},\end{cases}
$$

and if $\gamma=0$ we have

$$
c\left\|\sigma^{\frac{1}{2}} u_{d}\right\|_{\mathcal{K}}^{2} \leq\left|a_{s}\left(u_{d}, u_{d}\right)\right|
$$

on general meshes and for meshes described in Proposition 5.6 we have

$$
c\left|\left\|u_{d}\right\|_{d}^{2} \leq\right| a_{s}\left(u_{d}, u_{d}\right) \mid
$$

REMARK 8.7. Observe that the method is well posed also for $\gamma=0$. 
Proof. Let us prove first the well-posedness of (8.2) for $s \gamma<0$. Observe that

$$
\begin{aligned}
c \min (1,|\gamma|) \mid\left\|u_{d}\right\|_{d}^{2} & \leq\left\|\sigma^{\frac{1}{2}} \nabla u_{d}\right\|_{\mathcal{K}}^{2}+|\gamma|\left\|\omega^{\frac{1}{2}} \tilde{h}_{\mathcal{F}}^{-\frac{1}{2}} \overline{\left[u_{d}\right]}\right\|_{\mathcal{F}_{i}}^{2} \\
& =-s a_{s}\left(u_{d}, u_{d}\right)+|\gamma| j\left(u_{d}, u_{d}\right) \\
& =\left|a_{s}\left(u_{d}, u_{d}\right)+\gamma j\left(u_{d}, u_{d}\right)\right|
\end{aligned}
$$

applying Lemma 5.1 and since $\left\|\omega^{\frac{1}{2}} \tilde{h}_{\mathcal{F}}^{-\frac{1}{2}} \overline{\left[u_{d}\right]}\right\|_{\mathcal{F}_{e}}^{2}=0$.

For $s \gamma>0$ observe that using the inverse and trace inequalities yields

$$
\left\|u_{d}\right\|_{d}^{2}=\left\|\sigma^{\frac{1}{2}} \nabla u_{d}\right\|_{\mathcal{K}}^{2}+\left\|\omega^{\frac{1}{2}} \tilde{h}_{\mathcal{F}}^{-\frac{1}{2}}\left[u_{d}\right]\right\|_{\mathcal{F}}^{2} \leq c\left\|\tilde{h}^{-1} \sigma^{\frac{1}{2}} u_{d}\right\|_{\mathcal{K}}^{2}
$$

On the other hand by norm equivalence in discrete spaces there exists a constant $c_{\star}>0$, independent of the mesh size $h$, such that

$$
\left\|\omega^{\frac{1}{2}} \tilde{h}_{\mathcal{F}}^{-\frac{1}{2}} \overline{\left[u_{d}\right]}\right\|_{\mathcal{F}_{i}}^{2} \geq c_{\star}\left\|\tilde{h}^{-1} \sigma^{\frac{1}{2}} u_{d}\right\|_{\mathcal{K}}^{2},
$$

since $\left.\omega\right|_{F} \geq\left.\rho \sigma\right|_{\kappa_{i}}, i=1,2$. Thus using the inverse inequality with constant $c_{i e}$ yields

$$
\begin{aligned}
\left|a_{s}\left(u_{d}, u_{d}\right)+\gamma j\left(u_{d}, u_{d}\right)\right| & \geq-\left\|\sigma^{\frac{1}{2}} \nabla u_{d}\right\|_{\mathcal{K}}^{2}+s \gamma\left\|\omega^{\frac{1}{2}} \tilde{h}_{\mathcal{F}}^{-\frac{1}{2}} \overline{\left[u_{d}\right]}\right\|_{\mathcal{F}_{i}}^{2} \\
& \geq\left(s \gamma c_{\star}-c_{i e}\right)\left\|\tilde{h}^{-1} \sigma^{\frac{1}{2}} u_{d}\right\|_{\mathcal{K}}^{2} .
\end{aligned}
$$

Observe that coercivity holds under the assumption that $s \gamma=|\gamma|>\frac{c_{i e}}{c_{\star}}=: C_{\text {stab }}$.

For $\gamma=0$ on general meshes observe that

$$
c\left\|\sigma^{\frac{1}{2}} u_{d}\right\|_{\mathcal{K}}^{2} \leq\left\|\sigma^{\frac{1}{2}} \nabla u_{d}\right\|_{\mathcal{K}}^{2}=-s a_{s}\left(u_{d}, u_{d}\right)=\left|a_{s}\left(u_{d}, u_{d}\right)\right|
$$

using the Poincaré inequality, Proposition 5.5.

For $\gamma=0$ on meshes described in Proposition 5.6 we have

$$
\begin{aligned}
\left\|u_{d}\right\|_{d}^{2} & =\left\|\sigma^{\frac{1}{2}} \nabla u_{d}\right\|_{\mathcal{K}}^{2}+\left\|\omega^{\frac{1}{2}} \tilde{h}_{\mathcal{F}}^{-\frac{1}{2}}\left[u_{d}\right]\right\|_{\mathcal{F}}^{2} \leq\left\|\sigma^{\frac{1}{2}} \nabla u_{d}\right\|_{\mathcal{K}}^{2}+c\left\|\tilde{h}^{-1} \sigma^{\frac{1}{2}} u_{d}\right\|_{\mathcal{K}}^{2} \\
& \leq c\left\|\sigma^{\frac{1}{2}} \nabla u_{d}\right\|_{\mathcal{K}}^{2}=c\left|a_{s}\left(u_{d}, u_{d}\right)\right|
\end{aligned}
$$

using the trace inequality and the strong Poincaré inequality, Proposition 5.6.

8.2. Convergence. We will now address the question of optimal convergence for different values of the stabilization parameter. In the case where the stabilization parameter is set to zero the lack of continuity of the bilinear form may perturb convergence. However if the mesh has the macro element structure of Proposition 5.6 optimal convergence is recovered.

THEOREM 8.8. Let $u \in H^{2}(\Omega)$ be the solution of (2.1) and $u_{h}^{1}$ the solution of (6.2) with $s \gamma<0, s \gamma>C_{\text {stab }}$ or $\gamma=0$ on the meshes defined in Proposition 5.6, then there holds

$$
\left\|u-u_{h}^{1}\right\| \mid \leq c h\|u\|_{2, \mathcal{K}}
$$

REMARK 8.9. On general meshes, in the particular case $\gamma=0$, this theorem is no more valid. In this case an optimal convergence result can be shown under some restrictive regularity assumptions on $f$ and the mesh, see Theorem 8.10.

Proof. First note that for the bilinear form $a_{s}(\cdot, \cdot)$ the following continuity holds for all $w \in H^{1}(\Omega), w_{c} \in V^{C}$ and $v_{d} \in V^{D}$ by Cauchy - Schwarz inequality

$$
a_{s}\left(w-w_{c}, v_{d}\right) \leq\left\|\mid w-w_{c}\right\|\left\|_{c}\right\| v_{d} \|_{d} .
$$


1. Decompose the error in two midpoint-continuous parts and one midpointdiscontinuous part,

$$
\left\|u-u_{h}^{1}\right\||\leq|\left\|u_{c}-i_{c} u\right\||+|\left\|u-i_{c} u\right\||+|\left\|u_{d}\right\| \mid
$$

2. Observe that by (7.1), Lemma 8.3 and Lemma 6.3:

$$
\begin{aligned}
\left\|u_{c}-i_{c} u\right\|^{2} & \leq c \mid\left\|u_{c}-i_{c} u\right\| \|_{d}^{2} \leq c a_{s}\left(u_{c}-i_{c} u, i_{c} u-u_{c}\right) \\
& =c a_{s}\left(u-i_{c} u-u_{d}, i_{c} u-u_{c}\right) \\
& \leq c\left(\left\|u-i_{c} u\right\|+\left\|\left|u_{d} \|\right|\right) \mid\left\|u_{c}-i_{c} u\right\|,\right.
\end{aligned}
$$

since $u_{c}-i_{c} u+u-u_{h}^{1}=u-i_{c} u-u_{d}$ and $a_{s}\left(u-u_{h}^{1}, i_{c} u-u_{c}\right)=0$. Thus

$$
\left\|u-u_{h}^{1}\right\| \mid \leq c\left(\left|\left\|u-i_{c} u\right\|\right|+\left|\left\|u_{d} \mid\right\|\right) .\right.
$$

3. Use Lemma 7.2 to bound the first term of (8.8),

$$
\left\|u-i_{c} u\right\| \mid \leq \operatorname{ch}\|u\|_{2, \mathcal{K}} .
$$

4. For the second term of (8.8) use (7.1) and the coercivity, Lemma 8.6,

$$
\left\|u_{d}\right\|^{2} \leq c\left|\left\|u_{d}\right\|_{d}^{2} \leq \frac{c}{C(\gamma)}\right| a_{s}\left(u_{d}, u_{d}\right)+\gamma j\left(u_{d}, u_{d}\right) \mid .
$$

In the particular case $\gamma=0$, the constant $C(0)$ denotes the constant of $(8.5)$.

5. Use the consistency of the bilinear form, Lemma 6.3,

$$
\left\|u_{d}\right\|^{2} \leq \frac{c}{C(\gamma)}\left|a_{s}\left(u-u_{c}, u_{d}\right)\right|
$$

since $u_{d}+u-u_{h}^{1}=u-u_{c}$ and $a_{s}\left(u-u_{h}^{1}, u_{d}\right)-\gamma j\left(u_{h}^{1}, u_{d}\right)=0$.

6. Conclude by applying the continuity (8.6) and the approximation result (7.5).

Under some restrictions we can show optimal convergence also in the particular case of $\gamma=0$ for the symmetric version on meshes without the macro element property.

THEOREM 8.10. Let $u \in H^{2}(\Omega)$ be the solution of (2.1) and $u_{h}^{1}$ the solution of (6.2) with $\gamma=0$ and $s=1$. Assume that $f \in H^{\beta}(\Omega)$, with $\beta \in\{1,2\}$, and that the mesh is asymptotically uniform with some $\zeta>0$, then there holds

$$
\left\|\nabla\left(u-u_{h}^{1}\right)\right\|_{\mathcal{K}} \leq c\left(h\|u\|_{2, \mathcal{K}}+\left(h^{\zeta}+h^{2^{\star}}\right)\|f\|_{1, \Omega}+h^{\beta}\|f\|_{\beta, \mathcal{K}}\right),
$$

where the term marked by * appears only in the 3-dimensional case.

Proof. Using the triangle inequality we can split

$$
\left\|\nabla\left(u-u_{h}^{1}\right)\right\|_{\mathcal{K}} \leq\left\|\nabla\left(u-u_{c}\right)\right\|_{\mathcal{K}}+\left\|\nabla u_{d}\right\|_{\mathcal{K}}
$$

Since $u_{c}$ is the standard Crouzeix-Raviart solution the first term of the right hand side of the previous equation can be bounded by

$$
\left\|\nabla\left(u-u_{c}\right)\right\|_{\mathcal{K}} \leq c h\|u\|_{2, \mathcal{K}}
$$

From (8.2) we can write

$$
\left\|\nabla u_{d}\right\|^{2}=\left|\left(f, u_{d}\right)_{\mathcal{K}}\right| \leq\left|\left(f-i_{c} f, u_{d}\right)_{\mathcal{K}}\right|+\left|\left(i_{c} f, u_{d}\right)_{\mathcal{K}}\right|
$$


where $i_{c}$ is the Crouzeix-Raviart interpolant introduced in Remark 4.2. The first term of the right hand side of (8.9) can be bounded by

$$
\left|\left(f-i_{c} f, u_{d}\right)_{\mathcal{K}}\right| \leq\left\|f-i_{c} f\right\|_{\mathcal{K}}\left\|u_{d}\right\|_{\mathcal{K}} \leq c h^{\beta}\|f\|_{\beta, \mathcal{K}}\left\|\nabla u_{d}\right\|_{\mathcal{K}}
$$

by optimal approximation properties of the Couzeix-Raviart interpolant and by the Poincaré inequality for $V^{D}$, Proposition 5.5.

For the second term of the right hand side of (8.9) we use Lemma 4.3, the Poincaré inequality for $V^{D}$, Proposition 5.5, and the stability of the Crouzeix-Raviart interpolant $\left\|i_{c} f\right\|_{\mathcal{K}} \leq\|f\|_{1, \Omega}$ resp. $\left\|\nabla i_{c} f\right\|_{\mathcal{K}} \leq\|f\|_{1, \Omega}$ :

$$
\begin{aligned}
\left|\left(i_{c} f, u_{d}\right)_{\mathcal{K}}\right| & \leq c\left(h^{\zeta}\left\|i_{c} f\right\|_{\mathcal{K}}\left\|u_{d}\right\|_{\mathcal{K}}+h^{2^{\star}}\left\|\nabla i_{c} f\right\|_{\mathcal{K}}\left\|\nabla u_{d}\right\|_{\mathcal{K}}\right) \\
& \leq c\left(h^{\zeta}+h^{2}\right)\|f\|_{1, \Omega}\left\|\nabla u_{d}\right\|_{\mathcal{K}}
\end{aligned}
$$

The term marked by $\star$ appears only in the 3 -dimensional case. Combining (8.10) and (8.11) completes the proof. $\square$

REMARK 8.11. If $\beta=2$ and $\zeta=2$, then optimal convergence can be shown in the triple norm ||$\cdot|| \mid$ since in this case

$$
\left\|\omega^{\frac{1}{2}} \tilde{h}_{\mathcal{F}}^{-\frac{1}{2}}\left[u_{d}\right]\right\|_{\mathcal{F}}^{2} \leq c\left\|\tilde{h}^{-1} \nabla u_{d}\right\|_{\mathcal{K}} \leq c h\|f\|_{2, \mathcal{K}}
$$

REMARK 8.12. Observe that on uniform meshes the convergence is only limited by the regularity of $f$.

We will now show that we have optimal $L^{2}$-convergence for the symmetric version thanks to the adjoint consistency. For the non-symmetric version the $L^{2}$-convergence rate depends on the regularity of the mesh and the right hand side as pointed out in $[14]$ in the one dimensional case.

THEOREM 8.13. Let $u \in H^{2}(\Omega)$ with $\|u\|_{2, \Omega} \leq c\|f\|_{\Omega}$ be the solution of (2.1) and $u_{h}^{1}$ the solution of (6.2) with $s \gamma<0, s \gamma>C_{\text {stab }}$ or $\gamma=0$ on meshes as described in Proposition 5.6, then there holds:

a) If $s=1$, then

$$
\left\|u-u_{h}^{1}\right\|_{L^{2}(\Omega)} \leq c h^{2}\|u\|_{2, \Omega} .
$$

b) If $s=-1$, and assuming that $f \in H^{\beta}(\Omega)$, with $\beta \in\{1,2\}$, and that the mesh is assymptotically uniform with some $\zeta>0$, then

$$
\left\|u-u_{h}^{1}\right\|_{L^{2}(\Omega)} \leq c\left(h^{2}\|u\|_{2, \mathcal{K}}+\left(h^{\zeta}+h^{2^{\star}}\right)\|f\|_{1, \Omega}+h^{\beta}\|f\|_{\beta, \mathcal{K}}\right),
$$

where the term marked by $\star$ appears only in the 3-dimensional case.

Proof. Consider the dual problem: Find $\phi \in H_{0}^{1}(\Omega)$ such that

$$
(\sigma \nabla \phi, \nabla z)_{\Omega}=(e, z)_{\Omega}, \quad \forall z \in H_{0}^{1}(\Omega) .
$$

Under the regularity assumptions on $u$ we have $\|\phi\|_{1, \Omega} \leq c\|e\|_{\Omega}$ and $\|\phi\|_{2, \Omega} \leq c\|e\|_{\Omega}$. It follows that

$$
-(\nabla \cdot \sigma \nabla \phi, z)_{\Omega}=(e, z)_{\Omega}, \quad \forall z \in L^{2}(\Omega)
$$


Let $e=u-u_{h}$ then we have by the dual consistency of Lemma 6.3

$$
\begin{aligned}
\|e\|_{\Omega}^{2} & =a_{1}(e, \phi)=a_{s}(e, \phi)+(s-1)(\{\sigma \nabla \phi\},[e])_{\mathcal{F}_{i}} \\
& =a_{s}\left(e, \phi-i_{c} \phi\right)-(s-1)\left(\{\sigma \nabla \phi\},\left[u_{d}\right]\right)_{\mathcal{F}_{i}} \\
& \leq\|e\|\left\|_{d}\right\| \phi-i_{c} \phi\left\|_{c}+|s-1|\right\| \phi\left\|_{1, \mathcal{K}}^{\frac{1}{2}}\right\| \phi\left\|_{2, \mathcal{K}}^{\frac{1}{2}}\right\| u_{d} \| \\
& \leq c h\|e\|\left\|_{d}\right\| \phi\left\|_{2, \Omega}+|s-1|\right\| \phi\left\|_{1, \mathcal{K}}^{\frac{1}{2}}\right\| \phi\left\|_{2, \mathcal{K}}^{\frac{1}{2}}\right\| u_{d}\|\|
\end{aligned}
$$

by (8.6) and (7.5). We conclude using the a priori bound on the $H^{1}(\Omega)$ and $H^{2}(\Omega)$ norm of $\phi$ and Theorem 8.8,

$$
\|e\|_{\Omega} \leq c\left(h|\|e\||+|s-1|\left|\left\|u_{d}\right\|\right|\right) \leq c\left(h^{2}\|u\|_{2, \Omega}+|s-1|\left\|\left|u_{d}\right|\right\|\right) .
$$

For the symmetric case the result follows immediately since $|s-1|=0$.

Consider now the non-symmetric case for which $s=-1$. By (7.1) and the coercivity, Lemma 8.6, it follows that

$$
C(\gamma)\left|\left\|u_{d}\right\|\right|^{2} \leq C(\gamma)\left|\left\|u_{d}\left|\|_{d}^{2} \leq\right| a_{-1}\left(u_{d}, u_{d}\right)+\gamma j\left(u_{d}, u_{d}\right)|=|\left(f, u_{d}\right)_{\mathcal{K}} \mid .\right.\right.
$$

Then split $f$ into a midpoint continuous and discontinuous part and use Lemma 4.3, (7.3) and the stability of the Crouzeix-Raviart interpolant

$$
\begin{aligned}
\left.C(\gamma)\left\|u_{d}\right\|\right|^{2} & \leq\left|\left(f-i_{c} f, u_{d}\right)_{\mathcal{K}}\right|+\left|\left(i_{c} f, u_{d}\right)_{\mathcal{K}}\right| \\
& \leq\left(\left\|f-i_{c} f\right\|_{\mathcal{K}}+c h^{\zeta}\left\|i_{c} f\right\|_{\mathcal{K}}\right)\left\|u_{d}\right\|_{\mathcal{K}}+c h^{2^{\star}}\left\|\nabla i_{c} f\right\|_{\mathcal{K}}\left\|\nabla u_{d}\right\|_{\mathcal{K}} \\
& \leq\left(c h^{\beta}\|f\|_{\beta, \mathcal{K}}+c\left(h^{\zeta}+h^{2^{\star}}\right)\|f\|_{1, \Omega}\right)\left\|u_{d}\right\| .
\end{aligned}
$$

Thus the result in the non-symmetric case. $\square$

REMARK 8.14. For $\gamma=0$, in the particular case of Remark 8.11 optimal convergence in the $L^{2}$-norm can be shown also on regular meshes without the macroelement property. The details are left to the reader.

8.3. Numerical Tests. Observe that the only difference between the standard SIPG/NIPG-method and the method 1 is that in the latter case the stabilization term is composed by the facewise $L^{2}$-projection of order 0 of the jumps. From an implementational viewpoint this can be realized by reducing the order of the quadrature formula for the numerical integration on the faces, i.e. applying the midpoint integration rules for the computation of the stabilization term.

8.3.1. Test problems. Let us briefly present the test problems used for the numerical tests.

a) Problem with smooth solution

We consider problem (2.1) with $\sigma=1$ and $f=2\left(2-x^{2}-y^{2}\right)$ on the square $\Omega=$ $(-1,1)^{2}$. The exact solution is given by $u(x, y)=\left(x^{2}-1\right)\left(y^{2}-1\right) \in C^{\infty}(\Omega)$. A sequence of unstructured meshes is considered.

b) Problem with irregular solution

Now choose the following $L$-shaped domain: $\Omega=(-1,1) \times(-1,0) \cup(0,1)^{2}$. We consider problem (2.1) with $\sigma=1$ and $f=0$ and non-homogeneous boundary conditions such that the solution is

$$
u(x, y)=\left(x^{2}+y^{2}\right)^{\frac{1}{3}} \sin \left(\frac{2}{3} \arctan _{*}\left(\frac{x}{y}\right)\right)
$$

where $\arctan _{*}$ is chosen in the manner that it is a continuous function at points with $y=0$. One can prove that $u \notin H^{2}(\Omega)$. Therefore the Theorems 8.8, 8.13, 9.5 and 9.6 are no longer valid. A sequence of unstructured meshes is considered. 


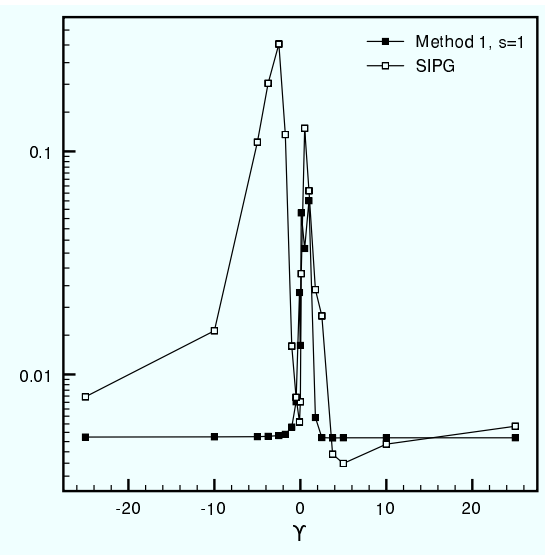

(a) $\left\|u-u_{h}\right\|_{\mathcal{K}}$

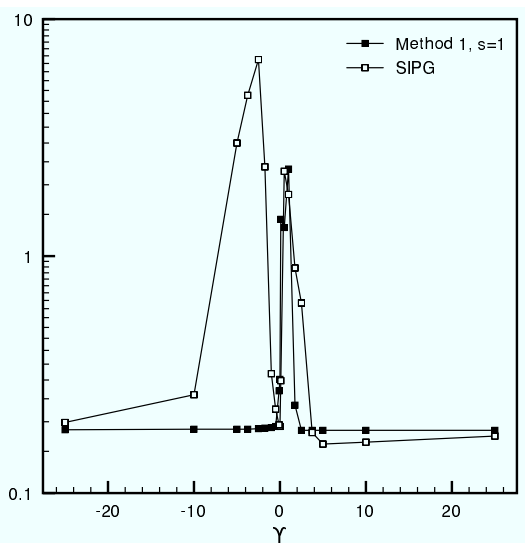

(b) $\left\|\nabla\left(u-u_{h}\right)\right\|_{\mathcal{K}}$

FIG. 8.1. Difference between the symmetric version of method 1 and the SIPG-method for the smooth problem measured in the $L^{2}$-error (a) resp. $H^{1}$-error (b) .

c) Problem with checkerboard mode

We consider problem (2.1) with $\sigma=1$ and $f=-1+2 \chi_{x>y}$ where $\chi$ denotes the characteristic function on the square $\Omega=(-1,1)^{2}$. A sequence of structured meshes is considered.

8.3.2. Robustness with respect to the stabilization parameter. Let us consider the problem with smooth solution. We compare the robustness of the symmetric version of method 1 with the SIPG-method respectively the non-symmetric version of method 1 with the NIPG-method.

Figure 8.1 illustrates the behavior of the error in the $L^{2}$ - and $H^{1}$-norm with varying stabilization parameter for the symmetric version of method 1 on a fixed mesh. In Figure 8.2 we present the same quantities for the non-symmetric case. Finally in Figure 8.3 we give comparisons of the condition number of the corresponding matrices. We define the condition number of a square matrix (not necessarily symmetric positive definite) as the ratio of the largest singular value of the matrix to the smallest one. Since the continuous and the discontinuous part of the approximation decouples for our formulation, we may also consider negative values of the penalization parameter.

8.3.3. Convergence. The convergence rates of method 1 with stabilization parameter $\gamma=0$ are compared to those of the standard SIPG- and NIPG-method once for the problem a) with regular solution and once for the problem b) with irregular solution.

Figure 8.4 shows the convergence rates of the smooth problem measured in the $L^{2}$ - and $H^{1}$-norm. The symmetric versions have slightly better convergence rates in the $L^{2}$-norm which can be justified by Theorem 8.13.

Figure 8.5 shows the accuracy of the methods for the problem with irregular solution. The SIPG-method has a much smaller $L^{2}$-error than the other methods, the convergence rates however seem to be the same.

The test problem $c$ ) is chosen so as to give rise a checkerboard mode in the 


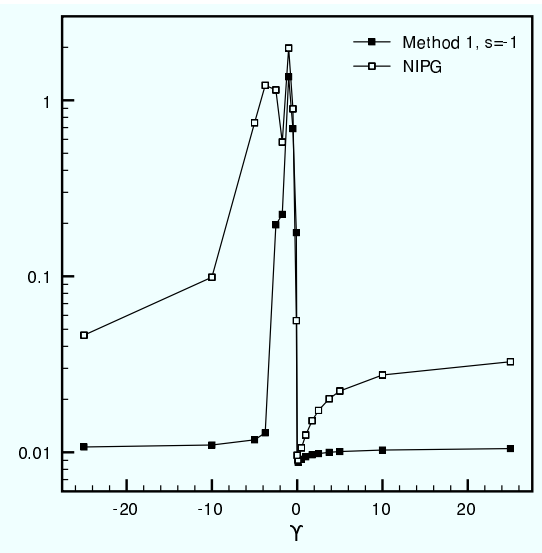

(a) $\left\|u-u_{h}\right\|_{\mathcal{K}}$

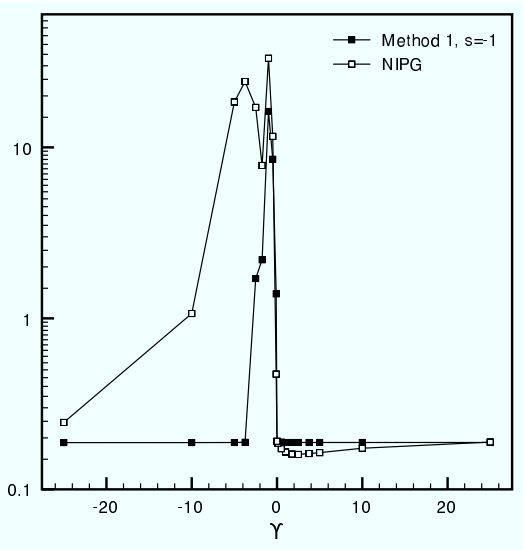

(b) $\left\|\nabla\left(u-u_{h}\right)\right\|_{\mathcal{K}}$

FIG. 8.2. Difference between the non-symmetric method 1 and the NIPG-method for the smooth problem measured in the $L^{2}$-error (a) resp. $H^{1}$-error (b) .

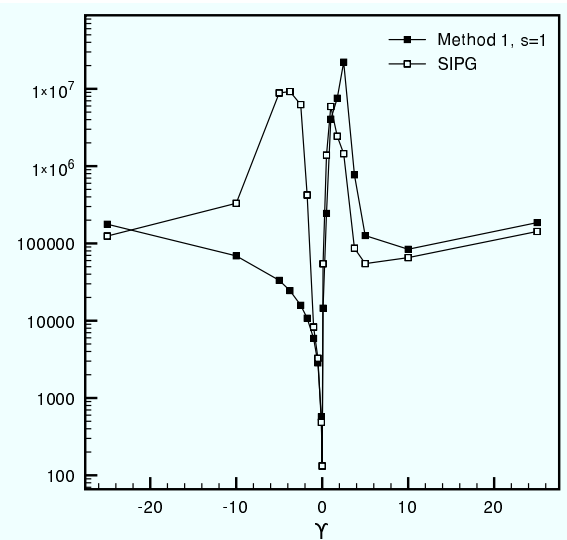

(a)

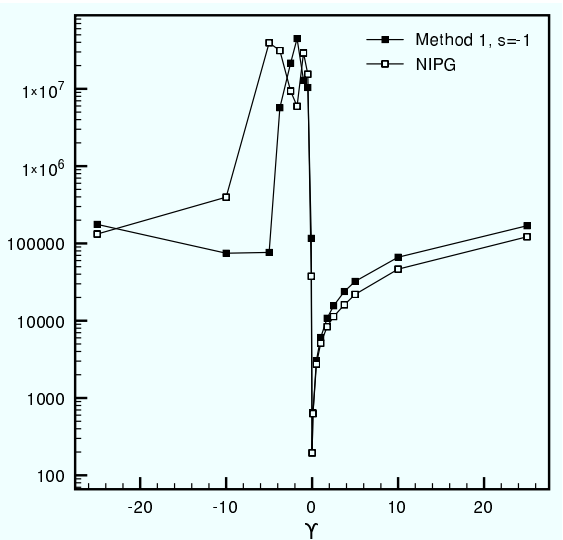

(b)

FIG. 8.3. Difference of condition number of matrices corresponding to the symmetric version (a) and non-symmetric version (b) of method 1 and the SIPG- (a) resp. NIPG-method (b) for the smooth problem.

discontinuous field. Here we give a convergence result for the symmetric version of method 1. The convergence of the jump term for different values of the stabilization parameter is given in Figure 8.6a and the convergence of the jump contribution in the broken $H^{1}$ semi-norm is given in Figure 8.6b. Clearly the broken $H^{1}$-norm of $u_{d}$ converges for the case without stabilization although the error does not converge in the norm $|\|\cdot\||$ including the jumps. This lack of convergence of the interelement jumps is caused by the checkerboard mode in the field $u_{d}$. In Figure 8.7 we give plots of the $u_{d}$ field for various values of the penalization parameter $\gamma$. This clearly illustrates how the penalization localizes the perturbation caused by the discontinuous data, and 


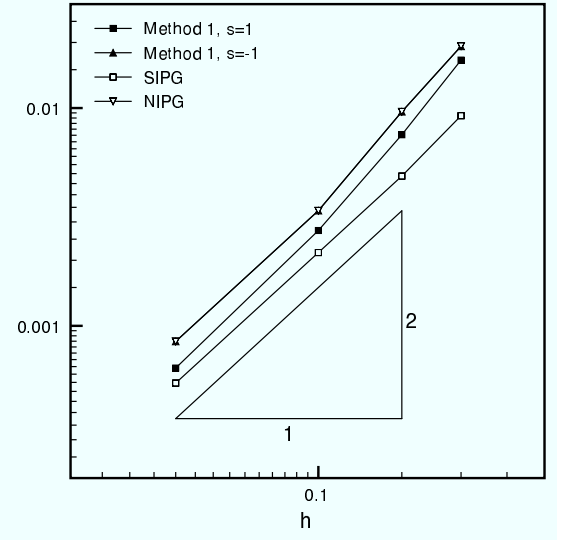

(a) $\left\|u-u_{h}\right\|_{\mathcal{K}}$

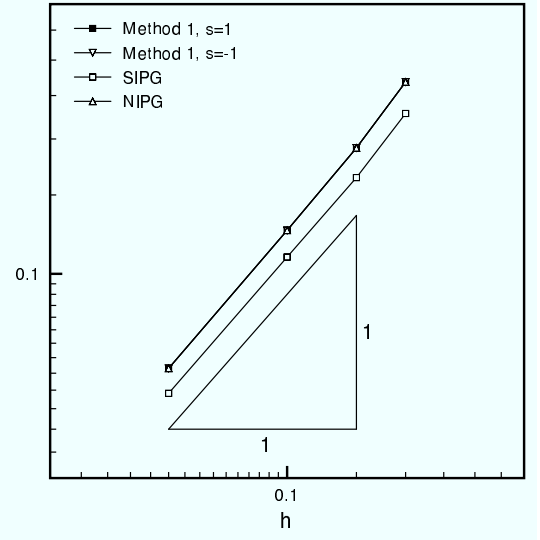

(b) $\left\|\nabla\left(u-u_{h}\right)\right\|_{\mathcal{K}}$

FIG. 8.4. $L^{2}$-error (a) and $H^{1}$-error (b) for $h$-refinement for the problem with smooth solution using stabilization parameters $\gamma=0$ for method $1, \gamma=10$ for the SIPG-method and $\gamma=1$ for the NIPG-method.

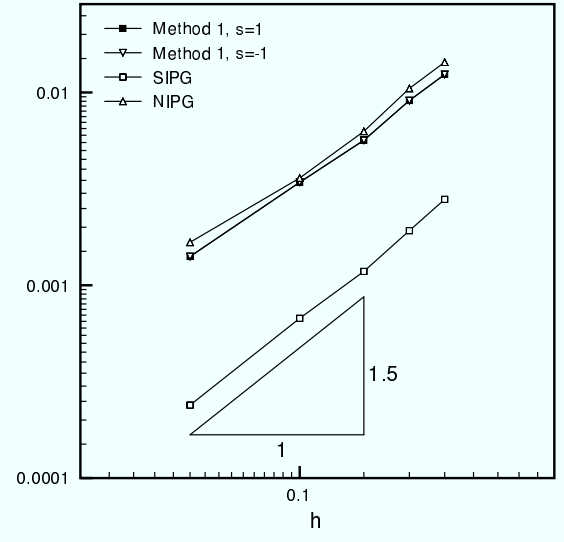

(a) $\left\|u-u_{h}\right\|_{\mathcal{K}}$

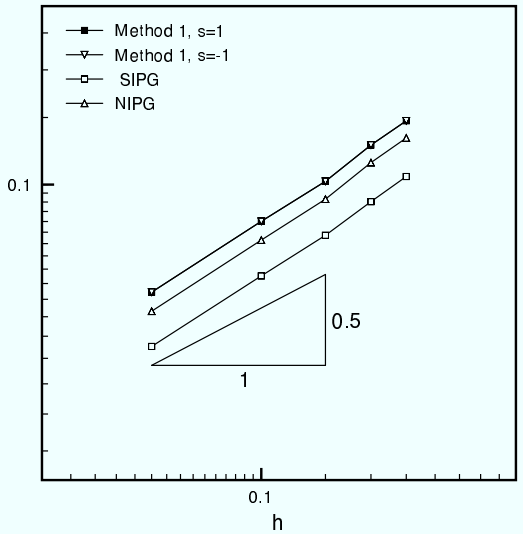

(b) $\left\|\nabla\left(u-u_{h}\right)\right\|_{\mathcal{K}}$

FIG. 8.5. $L^{2}$-error (a) and $H^{1}$-error (b) for $h$-refinement for the problem with irregular solution using stabilization parameters $\gamma=0$ for method $1, \gamma=10$ for the SIPG-method and $\gamma=1$ for the NIPG-method.

hence enhances convergence for $\gamma \neq 0$.

9. Analysis of method 2. In the previous section we saw that for the unstabilized symmetric DG method the appearance of a checkerboard mode for rough data destroyed convergence of the solution jumps. Optimal convergence is recovered if the mesh has a certain macro element structure. For method 2 this structure is replaced by a bubble enrichment of the space. The motivation for the DG-method using the enriched space is to obtain local mass conservation independent of the stabilization parameter for a low order DG-method while keeping optimal convergence properties 


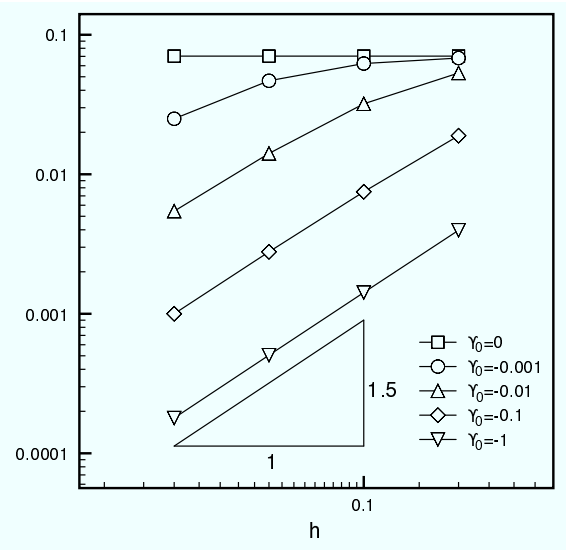

(a) $\left\|\tilde{h}_{\mathcal{F}}^{-\frac{1}{2}}\left[u_{d}\right]\right\|_{\mathcal{F}_{i}}$

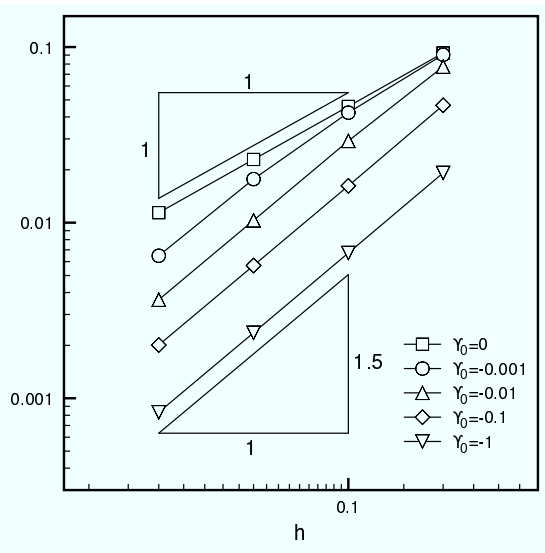

(b) $\left\|\nabla u_{d}\right\|_{\mathcal{K}}$

Fig. 8.6. Different norms of $u_{d}$ for $h$-refinement and for different stabilization parameters $\gamma$.

in the general case.

9.1. Projection. In order to prove stability of the method we first need to define the following projection.

LEMma 9.1 (Interpolant). Let $u_{h} \in V_{h}^{b}$ be a fixed function. Then there exists a unique $w_{h} \in V_{h}^{b}$ such that

$$
\begin{gathered}
\int_{\kappa} w_{h} d x=0 \quad \forall \kappa \in \mathcal{K} \\
\left.\left\{\sigma \nabla w_{h}\right\}\right|_{F} \cdot n_{F}=\omega h_{F}^{-1} \overline{\left[u_{h}\right]} \cdot n_{F} \quad \forall F \in \mathcal{F} \\
\int_{F}\left\{w_{h}\right\}=0 \quad \forall F \in \mathcal{F}_{i} .
\end{gathered}
$$

In addition the following a priori estimate holds

$$
\left\|\sigma^{\frac{1}{2}} \nabla w_{h}\right\|_{\mathcal{K}} \leq c\left\|\omega^{\frac{1}{2}} \tilde{h}_{\mathcal{F}}^{-\frac{1}{2}} \overline{\left[u_{h}\right]}\right\|_{\mathcal{F}},
$$

where $\omega=\min \left(\left.\sigma\right|_{\kappa_{1}},\left.\sigma\right|_{\kappa_{2}}\right)$ for $F=\partial \kappa_{1} \cap \partial \kappa_{2}$.

Proof. Let us first observe that the number of conditions on the projection is equal to the number of unknowns. The dimension of the finite element space $V_{h}^{b}$ is $(d+2) N_{e l}$ where $N_{e l}$ denotes the number of elements in the mesh. On the other hand condition (9.1) enforces $N_{e l}$ constraints whereas conditions (9.2) and (9.3) demand $N_{F}+N_{F_{i}}$ constraints where $N_{F}$ and $N_{F_{i}}$ denote the number of the number of faces resp. the number of interior faces of the mesh. Observing that $N_{F}+N_{F_{i}}=(d+1) N_{e l}$ implies directly a square linear system to determine the projection. Let us now establish the following a priori estimate

$$
\left\|w_{h}\right\||\leq c|\left|u_{h} \|\right| .
$$

Since $v_{h}$ has zero mean over each element it satisfies the following strong Poincaré inequality

$$
\left\|\sigma^{\frac{1}{2}} w_{h}\right\|_{\mathcal{K}} \leq c\left\|\tilde{h} \sigma^{\frac{1}{2}} \nabla w_{h}\right\|_{\mathcal{K}} .
$$




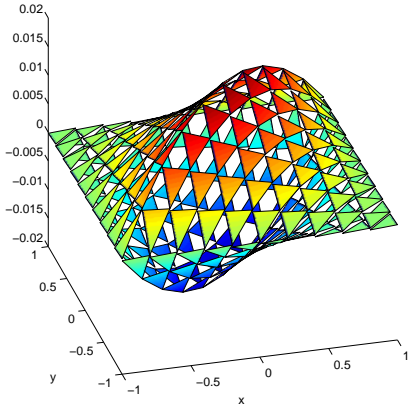

(a) $\gamma=0$

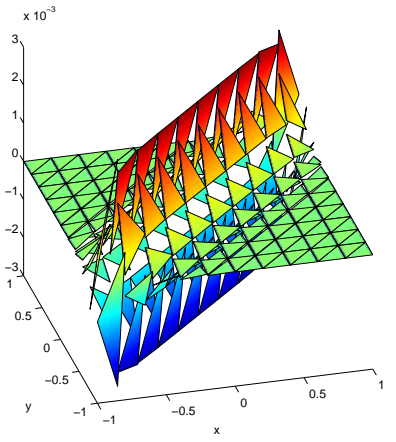

(c) $\gamma=-0.1$

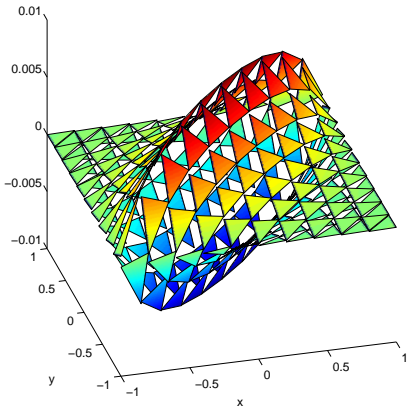

(b) $\gamma=-0.01$

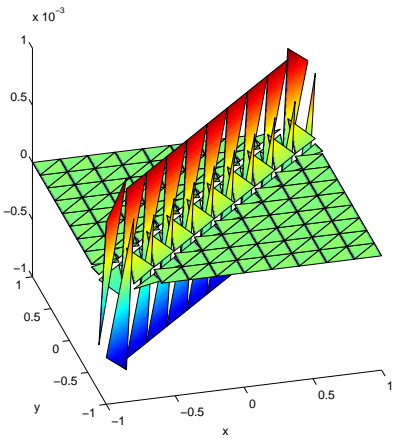

(d) $\gamma=-1$

FIG. 8.7. $u_{d}$ computed using different values of the stabilization parameter for the problem generating a checkerboard mode.

Hence, using a trace inequality yields

$$
\left\|\omega^{\frac{1}{2}} \tilde{h}_{\mathcal{F}}^{-\frac{1}{2}}\left[w_{h}\right]\right\|_{\mathcal{F}}^{2} \leq c\left\|\sigma^{\frac{1}{2}} \tilde{h}^{-1} w_{h}\right\|_{\mathcal{K}}^{2} \leq c\left\|\sigma^{\frac{1}{2}} \nabla w_{h}\right\|_{\mathcal{K}}^{2} .
$$

Moreover, integrating by parts and using the properties of $w_{h}$, it follows that

$$
\begin{aligned}
\left\|\sigma^{\frac{1}{2}} \nabla w_{h}\right\|_{\mathcal{K}}^{2} & =-\underbrace{\left(\nabla \cdot \sigma \nabla w_{h}, w_{h}\right)_{\mathcal{K}}}_{=0}+\left(\left\{\sigma \nabla w_{h}\right\},\left[w_{h}\right]\right)_{\mathcal{F}}+\underbrace{\left(\left[\sigma \nabla w_{h}\right],\left\{w_{h}\right\}\right)_{\mathcal{F}_{i}}}_{=0} \\
& =\left(\omega \tilde{h}_{\mathcal{F}}^{-1} \overline{\left[u_{h}\right]},\left[w_{h}\right]\right)_{\mathcal{F}} \leq\left\|\omega^{\frac{1}{2}} \tilde{h}_{\mathcal{F}}^{-\frac{1}{2}} \overline{\left[u_{h}\right]}\right\|_{\mathcal{F}}\left\|\omega^{\frac{1}{2}} \tilde{h}_{\mathcal{F}}^{-\frac{1}{2}}\left[w_{h}\right]\right\|_{\mathcal{F}}
\end{aligned}
$$

since $\left\{\sigma \nabla w_{h}\right\}$ and $\left[\sigma \nabla w_{h}\right]$ are constant along each face, see Lemma 4.4. Applying further the strong Poincaré inequality (9.6) proves (9.4)

$$
\left\|\sigma^{\frac{1}{2}} \nabla w_{h}\right\|_{\mathcal{K}} \leq c\left\|\omega^{\frac{1}{2}} \tilde{h}_{\mathcal{F}}^{-\frac{1}{2}} \overline{\left[u_{h}\right]}\right\|_{\mathcal{F}} .
$$

Additionally applying (7.1) and (9.7) implies that

$$
\left\|\mid w_{h}\right\| \leq \leq c\left\|\omega^{\frac{1}{2}} \tilde{h}_{\mathcal{F}}^{-\frac{1}{2}} \overline{\left[u_{h}\right]}\right\|_{\mathcal{F}} \leq\|\| u_{h}\|\| .
$$


Since we are faced to a square linear system, existence and uniqueness of a solution of the linear system are equivalent. Let us denote by $A w=F$ the square linear system and assume that there is a vector $w_{1}$ and $w_{2}$ such that $A w_{i}=F, i=1,2$. Further let us denote the difference between them by $e=w_{1}-w_{2}$ and therefore $A e=0$. The a priori estimate (9.5) implies that $e=0$ and thus uniqueness of the solution.

Corollary 9.2. Let $u_{h} \in V_{h}^{b}$ be a fixed function. Then there exists a unique $y_{h} \in V_{h}^{b}$ such that

$$
\begin{aligned}
\int_{\kappa} y_{h} d x & =0 \quad \forall \kappa \in \mathcal{K} \\
\left.\left\{\sigma \nabla y_{h}\right\}\right|_{F} \cdot n_{F} & =-s \omega h_{F}^{-1} \overline{\left[u_{h}\right]} \cdot n_{F} \quad \forall F \in \mathcal{F} \\
\int_{F}\left\{y_{h}\right\} & =\left[\sigma \nabla u_{h}\right] \quad \forall F \in \mathcal{F}_{i} .
\end{aligned}
$$

Proof. Since the matrix associated to the above defined projection $w_{h}$ has zero kernel, $y_{h}$ exists and is unique.

9.2. Stability. Although we do not explicitly penalize the solution jumps, control of the solution jumps in the triple norm is recovered by an inf-sup argument which is shown in this section.

LEMma 9.3. There is a constant $c>0$ independent of $h$ such that for all $u_{h} \in V_{h}^{b}$ there holds

$$
\left|\left(\left\{\sigma \nabla u_{h}\right\},\left[u_{h}\right]\right)_{\mathcal{F}}\right| \leq c\left\|\sigma^{\frac{1}{2}} \nabla u_{h}\right\|_{\mathcal{K}}\left\|\omega^{\frac{1}{2}} \tilde{h}_{\mathcal{F}}^{-\frac{1}{2}} \overline{\left[u_{h}\right]}\right\|_{\mathcal{F}}
$$

Proof. By a decomposition of the average operator we have that

$$
\left|\left(\left\{\sigma \nabla u_{h}\right\},\left[u_{h}\right]\right)_{\mathcal{F}}\right| \leq \sum_{\kappa \in \mathcal{K}} \frac{1}{2}\left|\left(\sigma \nabla u_{h},\left[u_{h}\right]\right)_{\partial \kappa}\right|,
$$

however since by definition $\nabla u_{h} \cdot n_{F} \in \mathbb{R}$ we have

$$
\left(\nabla u_{h},\left[u_{h}\right]\right)_{F}=\left(\nabla u_{h}, \overline{\left[u_{h}\right]}\right)_{F}, \quad \forall F \in \mathcal{F} .
$$

A Cauchy-Schwarz inequality and a trace inequality completes the proof noting that $\left.\sigma\right|_{\kappa_{1}} \leq\left.\rho \sigma\right|_{\kappa_{2}}$. $\mathrm{Q}$

THEOREM 9.4. (Discrete inf-sup condition) There exists a constant $c>0$ independent of $h$ such that for all $u_{h} \in V_{h}^{b}$ there holds

$$
c \mid\left\|u_{h}\right\| \leq \sup _{v_{h} \in V_{h}^{b}} \frac{a_{s}\left(u_{h}, v_{h}\right)}{\left\|v_{h}\right\| \mid}
$$

for $s \in\{-1,1\}$.

Proof. Let us prove this theorem in four steps:

Step 1: First we take $v_{h}=u_{h}$ in a standard fashion

$$
a_{s}\left(u_{h}, u_{h}\right)=\left\|\sigma^{\frac{1}{2}} \nabla u_{h}\right\|_{\mathcal{K}}^{2}-(1+s)\left(\left\{\sigma \nabla u_{h}\right\},\left[u_{h}\right]\right)_{\mathcal{F}} .
$$

Note that by Lemma 9.3 followed by an arithmetic-geometric inequality there exists a constant $c_{u}>0$ independent on the mesh size $h$ such that

$$
a_{s}\left(u_{h}, u_{h}\right) \geq \frac{1}{2}\left\|\sigma^{\frac{1}{2}} \nabla u_{h}\right\|_{\mathcal{K}}^{2}-c_{u}(1+s)^{2}\left\|\omega^{\frac{1}{2}} \tilde{h}_{\mathcal{F}}^{-\frac{1}{2}} \overline{\left[u_{h}\right]}\right\|_{\mathcal{F}}^{2} .
$$

Step 2: Second by Lemma 9.1 there exists $w_{h} \in V_{h}^{b}$ such that 
1. $\int_{\kappa} w_{h} \mathrm{~d} x=0$ for all $\kappa \in \mathcal{K}$,

2. $\left\{\sigma \nabla w_{h}\right\} \cdot n_{F}=\omega h_{F}^{-1} \overline{\left[u_{h}\right]} \cdot n_{F}$ on each face $F \in \mathcal{F}$,

3. $\int_{F}\left\{w_{h}\right\}=0$ on each face $F \in \mathcal{F}_{i}$.

An immediate consequence is that

$$
\begin{aligned}
a_{s}\left(u_{h}, w_{h}\right) & =-\left(\nabla \cdot \sigma \nabla u_{h}, w_{h}\right)_{\mathcal{K}}+\left(\left[\sigma \nabla u_{h}\right],\left\{w_{h}\right\}\right)_{\mathcal{F}_{i}}-s\left(\left\{\sigma \nabla w_{h}\right\},\left[u_{h}\right]\right)_{\mathcal{F}} \\
& =-s\left\|\omega^{\frac{1}{2}} \tilde{h}_{\mathcal{F}}^{-\frac{1}{2}} \overline{\left[u_{h}\right]}\right\|_{\mathcal{F}}^{2} .
\end{aligned}
$$

Step 3: Combining the results (9.8) and (9.9) we may take

$$
v_{h}=u_{h}-s\left(\frac{1}{2}+c_{u}(1+s)^{2}\right) w_{h}
$$

to obtain

$$
a_{s}\left(u_{h}, v_{h}\right) \geq \frac{1}{2} \mid\left\|u_{h}\right\|^{2}
$$

using Lemma 5.1 and Corollary 5.2.

Step 4: To conclude it remains to show that there exists $c>0$ independent of $h$ such that

$$
\left\|v_{h}\right\||\leq c|\left|u_{h} \|\right|
$$

This follows by straightforward estimation

$$
\left\|v_{h}\right\|\left|=\left\|u_{h}+c w_{h}\right\|\right| \leq\left\|u_{h}\right\| \mid+c\left\|w_{h}\right\| .
$$

Consider the second term of the right hand side after applying the strong Poincaré inequality (9.6)

$$
\left\|w_{h}\right\|^{2} \leq c(1+h)\left\|\sigma^{\frac{1}{2}} \nabla w_{h}\right\|_{\mathcal{K}}^{2}+\left\|\omega^{\frac{1}{2}} \tilde{h}_{\mathcal{F}}^{-\frac{1}{2}}\left[w_{h}\right]\right\|_{\mathcal{F}}^{2}=I_{1}+I_{2} .
$$

It follows by (9.4) that

$$
I_{1} \leq c\left\|\sigma^{\frac{1}{2}} \nabla w_{h}\right\|_{\mathcal{K}}^{2} \leq c\left\|\omega^{\frac{1}{2}} \tilde{h}_{\mathcal{F}}^{-\frac{1}{2}} \overline{\left[u_{h}\right]}\right\|_{\mathcal{F}}^{2},
$$

and by the trace inequality, the strong Poincaré inequality and by (9.4) that

$$
I_{2} \leq c\left\|\sigma^{\frac{1}{2}} \tilde{h}^{-1} w_{h}\right\|_{\mathcal{K}}^{2} \leq c\left\|\sigma^{\frac{1}{2}} \nabla w_{h}\right\|_{\mathcal{K}}^{2} \leq c\left\|\omega^{\frac{1}{2}} \tilde{h}_{\mathcal{F}}^{-\frac{1}{2}} \overline{\left[u_{h}\right]}\right\|_{\mathcal{F}}^{2} .
$$

Finally use that

$$
\left\|\omega^{\frac{1}{2}} \tilde{h}_{\mathcal{F}}^{-\frac{1}{2}} \overline{\left[u_{h}\right]}\right\|_{\mathcal{F}}^{2} \leq\left\|\omega^{\frac{1}{2}} \tilde{h}_{\mathcal{F}}^{-\frac{1}{2}}\left[u_{h}\right]\right\|_{\mathcal{F}}^{2}
$$

which completes the proof.

9.3. Convergence. Using the previously derived inf-sup condition optimal convergence is proved in a standard fashion.

THEOREM 9.5. Let $u \in H^{2}(\Omega)$ be the solution of (2.1) and $u_{h}^{b}$ the solution of (6.3) then there holds

$$
\left\|u-u_{h}^{b}\right\| \mid \leq c h\|u\|_{2, \mathcal{K}} .
$$

Proof. First note that for the bilinear form $a_{s}(\cdot, \cdot)$ the following continuity holds for all $v \in H^{1}(\mathcal{K})$ and $v_{h} \in V_{h}^{b}$ by Cauchy-Schwarz and trace inequality

$$
a_{s}\left(v, v_{h}\right) \leq\|v\|_{c}\left\|v_{h}\right\| .
$$


1. Decompose the error in a continuous and a discrete part.

$$
\left\|u-u_{h}^{b}\right\|\left|\leq\left\|u-\pi_{h} u\right\|\right|+\left\|\pi_{h} u-u_{h}^{b}\right\| \mid .
$$

Recall that $\pi_{h}$ denotes the element-wise $L^{2}$-projection onto $V_{h}^{1}$ and observe that the convergence of the continuous part follows by (7.2).

2. Use the inf-sup condition on the discrete part and the consistency of the bilinear form, Lemma 6.3

$$
c\left|\left\|\pi_{h} u-u_{h}^{b}\right\|\right| \leq \sup _{v_{h} \in V_{h}^{b}} \frac{a_{s}\left(\pi_{h} u-u_{h}^{b}, v_{h}\right)}{\left\|v_{h}\right\| \mid} \leq \sup _{v_{h} \in V_{h}^{b}} \frac{a_{s}\left(\pi_{h} u-u, v_{h}\right)}{\left\|v_{h}\right\| \mid} .
$$

3. Conclude by applying the continuity (9.10) and the approximation result $(7.2)$

We will now show that we have optimal $L^{2}$-convergence for the symmetric version thanks to the adjoint consistency.

THEOREM 9.6. Let $u \in H^{2}(\Omega)$ with $\|u\|_{2, \Omega} \leq c\|f\|_{\Omega}$ be the solution of (2.1) and $u_{h}^{b}$ the solution of (6.3) with $s=1$, then there holds

$$
\left\|u-u_{h}^{b}\right\|_{L^{2}(\Omega)} \leq c h^{2}\|u\|_{2, \Omega} .
$$

Proof. Consider the dual problem: Find $\phi \in H_{0}^{1}(\Omega)$ such that

$$
(\sigma \nabla \phi, \nabla z)_{\Omega}=(e, z)_{\Omega}, \quad \forall z \in H_{0}^{1}(\Omega) .
$$

Under the regularity assumptions on $u$ we have $\|\phi\|_{2, \Omega} \leq c\|e\|_{\Omega}$. It follows that

$$
-(\nabla \cdot \sigma \nabla \phi, z)_{\Omega}=(e, z)_{\Omega}, \quad \forall z \in L^{2}(\Omega)
$$

Let $e=u-u_{h}^{b}$ then we have by the dual consistency of Lemma 6.3

$$
\|e\|_{\Omega}^{2}=a_{1}(e, \phi)=a_{1}\left(e, \phi-\pi_{h} \phi\right) \leq\left\|\left|\left\|\left|\left\|\phi-\pi_{h} \phi\right\|\right| \leq c h\right\|\right|\right\|\|\| \phi \|_{2, \Omega}
$$

and we conclude using the a priori bound on the $H^{2}(\Omega)$ norm of $\phi$ and Theorem 9.5,

$$
\|e\|_{\Omega} \leq c h\|e\| \mid \leq c h^{2}\|u\|_{2, \Omega} .
$$

Additionally this method has some interesting properties as pointed out in the following remarks.

REMARK 9.7. Let $u_{h}^{b} \in V_{h}^{b}$ be the solution of (6.3). If the right hand side $f$ is piecewise constant, then there holds

$$
\left\|\left[\sigma \nabla u_{h}^{b}\right]\right\|_{\mathcal{F}_{i}}^{2}+\left\|\omega^{\frac{1}{2}} \tilde{h}_{\mathcal{F}}^{-\frac{1}{2}} \overline{\left[u_{h}^{b}\right]}\right\|_{\mathcal{F}}^{2}=0 .
$$

Indeed choosing the function $y_{h}$ defined in Corollary 9.2 in (6.3) and applying an integration by parts leads to the result.

9.4. Numerical Tests. We consider the same three test problems as in Section §8.3.1. In the case of method 2 the local mass conservation property is satisfied independently of the stabilization parameter. 


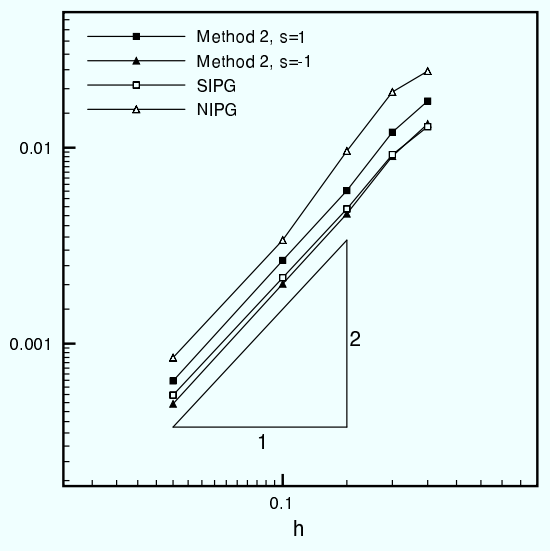

(a) $\left\|u-u_{h}\right\|_{\mathcal{K}}$

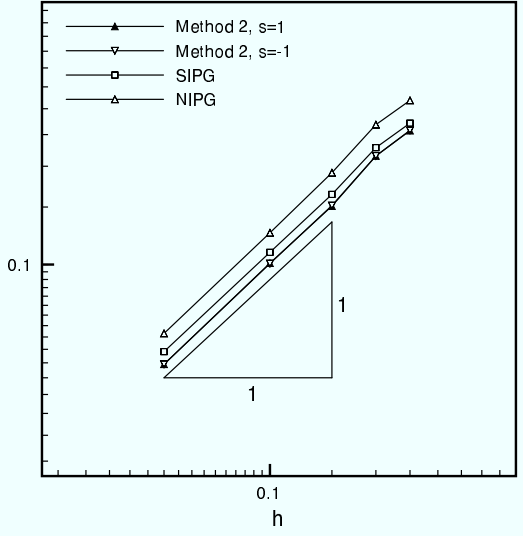

(b) $\left\|\nabla\left(u-u_{h}\right)\right\|_{\mathcal{K}}$

FIG. 9.1. $L^{2}$-error (a) and $H^{1}$-error (b) for $h$-refinement for the problem with smooth solution. For the SIPG- and NIPG-method a stablization parameter of $\gamma=10$ resp. $\gamma=1$ is chosen.

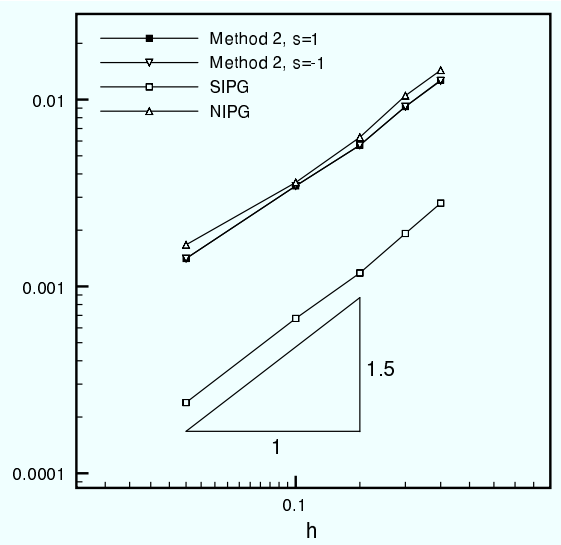

(a) $\left\|u-u_{h}\right\|_{\mathcal{K}}$

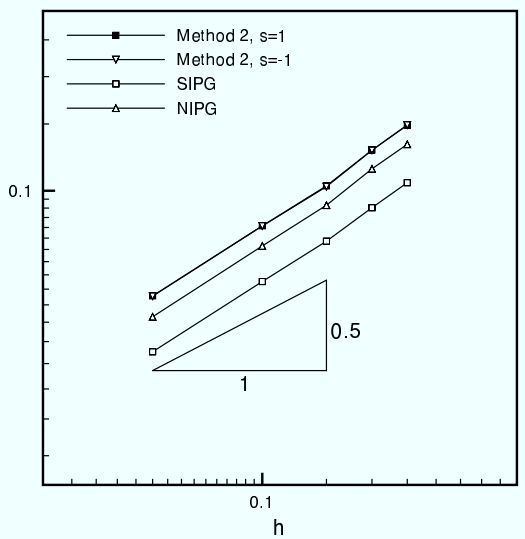

(b) $\left\|\nabla\left(u-u_{h}\right)\right\|_{\mathcal{K}}$

FIG. 9.2. $L^{2}$-error (a) and $H^{1}$-error (b) for $h$-refinement for the problem with smooth solution. For the SIPG- and NIPG-method a stablization parameter of $\gamma=10$ resp. $\gamma=1$ is chosen.

9.4.1. Convergence. In this section we give the convergence rates of method 2 for test problems a) and b). We get optimal convergence of the error in $H^{1}$ - and $L^{2}$ norms, and similar convergence curves as for SIPG and NIPG for the case of smooth exact solution (Figure 9.1). When the solution presents a singularity we once again observe a larger constant for method 2 than SIPG, in particular in the $L^{2}$-norm.

10. Conclusion. In this paper we discussed low order discontinuous Galerkin methods for second order scalar elliptic problems in two and three space dimensions. The main results are given in the following points:

(i) Midpoint imposition of Dirichlet boundary conditions is sufficient to assure 
discrete well-posedness for the symmetric DG-formulation using piecewise affine approximation (no interior stabilization is needed).

(ii) The linear system resulting from method 1 can be transformed into a system with reduced couplings between the continuous and discontinuous spaces. In the symmetric case $(s=1)$ the matrix is block diagonal and can be solved in parallel. In the non-symmetric case $(s=-1)$ the resulting matrix is upper triangular.

(iii) The symmetric version of method 1 without stabilization has optimal convergence in the broken $H^{1}$-norm and in the $L^{2}$-norm provided the meshes and data are sufficiently regular or satisfy the macro element property of Lemma 5.6.

(iv) The non-symmetric version of method 1 with stabilization has optimal convergence in the $L^{2}$-norm provided the meshes and data are sufficiently regular.

(v) For irregular data a checkerboard mode destroys convergence for the unstabilized DG-method when using piecewise affine approximations.

(vi) Enriching the space with non-conforming quadratic bubbles allows for a DG-method where the symmetric and non-symmetric versions are stable without stabilization, yielding optimal convergence in the broken $H^{1}$-norm and are locally mass conservative independently of the penalty parameter.

(vii) The symmetric DG-method on the enriched space has additionally optimal convergence in the $L^{2}$-norm.

The aim of this work was to construct a symmetric (i.e. adjoint consistent) DGmethod that enjoys optimal convergence and local mass conservation independently of the penalty parameter. This goal has been realized in the framework of low order approximation in the symmetric version of method 2 (see equation (6.3)) or, in the symmetric version of method 1 without penalization on interior faces when the mesh has the macro element property of Lemma 5.6.

Appendix. Here we detail the proof of the Poincaré inequality for the part $\mathcal{K}_{2}$ of the mesh in Proposition 5.5.

There exists a function $p \in H^{1}\left(\Omega_{2}^{j}\right)$ such that $\nabla \cdot p=\left|u_{d}\right|$ and $\|p\|_{1, \mathcal{K}_{2}^{j}} \leq c\left\|u_{d}\right\|_{0, \Omega_{2}^{j}}$. It follows that

$$
\begin{aligned}
\left\|u_{d}\right\|_{0, \Omega_{2}^{j}}^{2} & =\left(\left|u_{d}\right|,\left|u_{d}\right|\right)_{0, \Omega_{2}^{j}}=-\left(\nabla\left|u_{d}\right|, p\right)_{0, \Omega_{2}^{j}}+([|u|], p)_{\mathcal{F}_{i}\left(\mathcal{K}_{2}^{j}\right)}+\left(|u| \cdot n_{e}, p\right)_{\mathcal{F}_{e}\left(\mathcal{K}_{2}^{j}\right)} \\
& =I+I I+I I I
\end{aligned}
$$

where $n_{e}$ denotes the exterior normal with respect to $\mathcal{K}_{2}^{j}$. Let us develop each term separately. Using a Cauchy-Schwarz inequality yields directly

$$
I=-\left(\left|\nabla u_{d}\right|, p\right)_{0, \Omega_{2}^{j}} \leq\left\|\nabla u_{d}\right\|_{\mathcal{K}_{2}^{j}}\|p\|_{\mathcal{K}_{2}^{j}}
$$

since $u_{d} \neq 0, \forall x \in \Omega_{2}^{j}$. Using the the property that $\left|u_{d}\right|$ lies in the Crouzeix-Raviart space over $\mathcal{K}_{2}^{j}$, i.e. the midpoint conitinuity, yields

$$
\begin{aligned}
I I & =([|u|], p-\bar{p})_{\mathcal{F}_{i}\left(\mathcal{K}_{2}^{j}\right)}=([|u|-\bar{u}], p-\bar{p})_{\mathcal{F}_{i}\left(\mathcal{K}_{2}^{j}\right)} \leq \sum_{F \in \mathcal{F}_{i}\left(\mathcal{K}_{2}^{j}\right)}\|[u-\bar{u}]\|_{F}\|p-\bar{p}\|_{F} \\
& \leq c h|u|_{1, \mathcal{K}_{2}^{j}}|p|_{1, \mathcal{K}_{2}^{j}},
\end{aligned}
$$

where

$$
\bar{p}=\frac{1}{\operatorname{meas}(F)} \int_{F} p d s \quad \text { and } \quad \bar{q}=\frac{1}{\operatorname{meas}(F)} \int_{F} q d s, \quad \forall F \in \mathcal{F}_{i}\left(\mathcal{K}_{2}^{j}\right) .
$$


For the third term of (A.1) take an arbitrary face $F \in \mathcal{F}_{e}\left(\mathcal{K}_{2}^{j}\right)$ of the sum. Observe that the boundary of $\partial \Omega \subset \partial \Omega_{1}$ where

$$
\Omega_{1}=\left(\bigcup_{\kappa \in \mathcal{K}_{1}}^{\circ} \kappa\right)
$$

As consequence none of the faces of $\Omega_{2}^{j}$ are contained in the boundary of $\Omega$ and thus $\Omega_{2}^{j}$ shares the boundary with $\Omega_{1}$. Therefore write $F=\partial \kappa_{1}(F) \cap \partial \kappa_{2}(F)$ with $\kappa_{1}(F) \in \mathcal{K}_{1}$ and $\kappa_{2}(F) \in \mathcal{K}_{2}^{j}$. Let us denote $\kappa_{1}(F), \kappa_{2}(F)$ by $\kappa_{1}$ resp. $\kappa_{2}$ in the case that the face $F$ is fixed. Use again

$$
Z\left(\kappa_{1}\right)=\left\{x \in \kappa_{1}: u_{d}(x)=0\right\}
$$

and we can write again

$$
\left.u_{d}(x)\right|_{\kappa_{1}}=\left.\nabla u_{d}\right|_{\kappa_{1}} \cdot\left(x-x^{\star}\right) \quad \text { with } x^{\star} \in Z\left(\kappa_{1}\right), \quad \forall x \in \kappa_{1} .
$$

Let $x_{F}$ be the midpoint of the face $F$. By property of the space $V^{D}$ we have that

$$
0=2\left\{u_{d}\right\}\left(x_{F}\right)=\left.u_{d}\right|_{\kappa_{1}}\left(x_{F}\right)+\left.u_{d}\right|_{\kappa_{2}}\left(x_{F}\right)=\left.\nabla u_{d}\right|_{\kappa_{1}} \cdot\left(x_{F}-x^{\star}\right)+\left.u_{d}\right|_{\kappa_{2}}\left(x_{F}\right) .
$$

Thus

$\left.u_{d}\right|_{\kappa_{2}}(x)=\left.u_{d}\right|_{\kappa_{2}}\left(x_{F}\right)+\left.\nabla u_{d}\right|_{\kappa_{2}} \cdot\left(x-x_{F}\right)=-\left.\nabla u_{d}\right|_{\kappa_{1}} \cdot\left(x_{F}-x^{\star}\right)+\left.\nabla u_{d}\right|_{\kappa_{2}} \cdot\left(x-x_{F}\right)$

for all $x \in \kappa_{2}$. Then

$$
\begin{aligned}
\left|\left(|u| \cdot n_{e}, p\right)_{F}\right| & \leq\left|\left(h_{\kappa_{1}}\left|\nabla u_{d}\right|_{\kappa_{1}},|p|\right)_{F}+\left(h_{\kappa_{2}}\left|\nabla u_{d}\right|_{\kappa_{2}},|p|\right)_{F}\right| \\
& \leq\|p\|_{F}\left(\left\|\left.h_{\kappa_{1}} \nabla u_{d}\right|_{\kappa_{1}}\right\|_{F}+\left\|\left.h_{\kappa_{2}} \nabla u_{d}\right|_{\kappa_{2}}\right\|_{F}\right) \\
& \leq c_{T}\left(h_{\kappa_{2}}^{-\frac{1}{2}}\|p\|_{0, \kappa_{2}}+h_{\kappa_{2}}^{\frac{1}{2}}|p|_{1, \kappa_{2}}\right) \sum_{i=1}^{2}\left\|h_{\kappa_{i}}^{\frac{1}{2}} \nabla u_{d}\right\|_{\kappa_{i}}
\end{aligned}
$$

so that if $h_{\kappa_{1}} \leq \rho h_{\kappa_{2}}$ and $h_{\kappa_{2}} \leq 1$, then

$$
\left|\left(|u| \cdot n_{e}, p\right)_{F}\right| \leq c\left(\|p\|_{0, \kappa_{2}}+h_{\kappa_{2}}|p|_{1, \kappa_{2}}\right) \sum_{i=1}^{2}\left\|\nabla u_{d}\right\|_{\kappa_{i}} \leq c\|p\|_{1, \kappa_{2}} \sum_{i=1}^{2}\left\|\nabla u_{d}\right\|_{\kappa_{i}} .
$$

Define the boundary layer between $\mathcal{K}_{1}$ and the $\mathcal{K}_{2}^{j}$ by

$$
\begin{aligned}
& \mathcal{K}^{\Gamma, j}=\mathcal{K}_{1}^{\Gamma, j} \cap \mathcal{K}_{2}^{\Gamma, j}, \\
& \mathcal{K}_{1}^{\Gamma, j}=\left\{\kappa_{1} \in \mathcal{K}_{1}: \exists \kappa_{2} \in \mathcal{K}_{2}^{j} \text { s.t. } \partial \kappa_{1} \cap \partial \kappa_{2} \neq \emptyset\right\}, \\
& \mathcal{K}_{2}^{\Gamma, j}=\left\{\kappa_{2} \in \mathcal{K}_{2}^{j}: \exists \kappa_{1} \in \mathcal{K}_{1} \text { s.t. } \partial \kappa_{1} \cap \partial \kappa_{2} \neq \emptyset\right\} .
\end{aligned}
$$

Then

$$
\begin{aligned}
|I I I| & \leq \sum_{F \in \mathcal{F}_{e}\left(\mathcal{K}_{2}^{j}\right)}\left|\left(|u| \cdot n_{e}, p\right)_{F}\right| \leq c \sum_{F \in \mathcal{F}_{e}\left(\mathcal{K}_{2}^{j}\right)}\|p\|_{1, \kappa_{2}(F)}\left(\left\|\nabla u_{d}\right\|_{\kappa_{1}(F)}+\left\|\nabla u_{d}\right\|_{\kappa_{2}(F)}\right) \\
& \leq c\|p\|_{1, \mathcal{K}_{2}^{j}}\left\|\nabla u_{d}\right\|_{\mathcal{K}^{\Gamma, j}} \leq c\left\|u_{d}\right\|_{\mathcal{K}_{2}^{j}}\left\|\nabla u_{d}\right\|_{\mathcal{K}^{\Gamma, j}} .
\end{aligned}
$$


On the other hand using similar arguments as before

$$
\begin{aligned}
I+I I & \leq c\left\|\nabla u_{d}\right\|_{\mathcal{K}_{2}^{j}}\left(\|p\|_{\mathcal{K}_{2}^{j}}+h|p|_{1, \mathcal{K}_{2}^{j}}\right) \leq c\left\|\nabla u_{d}\right\|_{\mathcal{K}_{2}^{j}}\|p\|_{1, \mathcal{K}_{2}^{j}} \\
& \leq c\left\|\nabla u_{d}\right\|_{\mathcal{K}_{2}^{j}}\left\|u_{d}\right\|_{\mathcal{K}_{2}^{j}} .
\end{aligned}
$$

Thus

$$
\left\|u_{d}\right\|_{\Omega_{2}^{j}} \leq c\left(\left\|\nabla u_{d}\right\|_{\mathcal{K}_{2}^{j}}+\left\|\nabla u_{d}\right\|_{\mathcal{K}_{1}^{\Gamma, j}}\right)
$$

and

$$
\left\|u_{d}\right\|_{\Omega_{2}}^{2} \leq c\left(\left\|\nabla u_{d}\right\|_{\mathcal{K}_{2}}^{2}+\sum_{j=1}^{m}\left\|\nabla u_{d}\right\|_{\mathcal{K}_{1}^{\Gamma, j}}^{2}\right) .
$$

Observe that

$$
\sum_{j=1}^{m}\left\|\nabla u_{d}\right\|_{\mathcal{K}_{1}^{\Gamma, j}}^{2} \leq(d+1)\left\|\nabla u_{d}\right\|_{\mathcal{K}_{1}}^{2}
$$

since in the worst case an element $\kappa_{1} \in \mathcal{K}_{1}$ is at most in $(d+1)$ different $\mathcal{K}_{1}^{\Gamma, j}$ and thus

$$
\left\|u_{d}\right\|_{\Omega_{2}} \leq c\left\|\nabla u_{d}\right\|_{\mathcal{K}}
$$

\section{REFERENCES}

[1] D. N. ARnold, An interior penalty finite element method with discontinuous elements, SIAM J. Numer. Anal., 19 (1982), pp. 742-760.

[2] G. A. BAKER, Finite element methods for elliptic equations using nonconforming elements, Math. Comp., 31 (1977), pp. 45-59.

[3] F. BASSi And S. ReBAy, A high-order accurate discontinuous finite element method for the numerical solution of the compressible Navier-Stokes equations, J. Comput. Phys., 131 (1997), pp. 267-279.

[4] S. C. Brenner, Poincaré-Friedrichs inequalities for piecewise $H^{1}$ functions, SIAM J. Numer. Anal., 41 (2003), pp. 306-324 (electronic).

[5] F. Brezzi, B. Cockburn, L. D. Marini, And E. Süli, Stabilization mechanisms in discontinuous Galerkin finite element methods, Comput. Methods Appl. Mech. Engrg., 195 (2006), pp. 3293-3310.

[6] E. Burman And B. Stamm, Minimal stabilization of discontinuous Galerkin finite element methods for hyperbolic problems, J. Sci. Comput., (to appear).

[7] P. Castillo, B. Cockburn, I. Perugia, and D. Schötzau, An a priori error analysis of the local discontinuous Galerkin method for elliptic problems, SIAM J. Numer. Anal., 38 (2000), pp. 1676-1706 (electronic).

[8] B. Cockburn and C.-W. Shu, The local discontinuous Galerkin method for time-dependent convection-diffusion systems, SIAM J. Numer. Anal., 35 (1998), pp. 2440-2463 (electronic).

[9] M. CrouzeiX AND P.-A. RaviarT, Conforming and nonconforming finite element methods for solving the stationary Stokes equations. I, Rev. Française Automat. Informat. Recherche Opérationnelle Sér. Rouge, 7 (1973), pp. 33-75.

[10] J. Douglas And T. Dupont, Interior penalty procedures for elliptic and parabolic Galerkin methods, in Computing methods in applied sciences (Second Internat. Sympos., Versailles, 1975), Springer, Berlin, 1976, pp. 207-216. Lecture Notes in Phys., Vol. 58.

[11] A. Ern And J.-L. Guermond, Theory and Practice of Finite Elements, vol. 159 of Applied Mathematical Sciences, Springer-Verlag, New York, NY, 2004. 
[12] — Discontinuous Galerkin methods for Friedrichs' systems. i. general theory, SIAM J. Numer. Anal., 44 (2006), pp. 753-778.

[13] M. G. Larson And A. J. NikLasson, Conservation properties for the continuous and discontinuous Galerkin methods, Tech. Report 2000-08, Chalmers finite element center, 2000.

[14] — Analysis of a family of discontinuous Galerkin methods for elliptic problems: the one dimensional case, Numer. Math., 99 (2004), pp. 113-130.

[15] —- Analysis of a nonsymmetric discontinuous Galerkin method for elliptic problems: stability and energy error estimates, SIAM J. Numer. Anal., 42 (2004), pp. 252-264 (electronic).

[16] D. Marazzina, Mixed discontinuous Galerkin methods with minimal stabilization, in Numerical mathematics and advanced applications, Bermudez et al., ed., Springer, 2006.

[17] J. T. Oden, I. BabušKa, And C. E. Baumann, A discontinuous hp finite element method for diffusion problems, J. Comput. Phys., 146 (1998), pp. 491-519.

[18] B. Riviṫre, M. F. Wheeler, and V. Girault, A priori error estimates for finite element methods based on discontinuous approximation spaces for elliptic problems, SIAM J. Numer. Anal., 39 (2001), pp. 902-931 (electronic).

[19] A. Romkes, S. Prudhomme, And J. T. Oden, A priori error analyses of a stabilized discontinuous Galerkin method, Comput. Math. Appl., 46 (2003), pp. 1289-1311.

[20] S. J. Sherwin, R. M. Kirby, J. Peiró, R. L. Taylor, and O. C. Zienkiewicz, On $2 D$ elliptic discontinuous Galerkin methods, Internat. J. Numer. Methods Engrg., 65 (2006), pp. $752-784$.

[21] R. Temam, Navier-Stokes equations, AMS Chelsea Publishing, Providence, RI, 2001. Theory and numerical analysis, Reprint of the 1984 edition.

[22] M. F. WheELER, An elliptic collocation-finite element method with interior penalties, SIAM J. Numer. Anal., 15 (1978), pp. 152-161. 